\title{
ON AN OPEN QUESTION OF MOUDAFI FOR CONVEX FEASIBILITY PROBLEMS IN HILBERT SPACES
}

\author{
Eskandar Naraghirad
}

\begin{abstract}
Very recently, Moudafi (Nonlinear Analysis 79 (2013) 117-121) introduced a relaxed alternating CQ-algorithm (RACQA) with weak convergence for the following convex feasibility problem:

Find $x \in C, y \in Q \quad$ such that $A x=B y$,

where $H_{1}, H_{2}, H_{3}$ are real Hilbert spaces, $C \subset H_{1}, Q \subset H_{2}$ are two nonempty, closed and convex level sets, and $A: H_{1} \rightarrow H_{3}, B: H_{2} \rightarrow H_{3}$ are two bounded linear operators. In this paper, we will continue to consider the problem (1.1) and obtain a strongly convergent iterative sequence of Halpern-type to a solution of the problem and provide an affirmative answer to an open question posed by Moudafi in his recent work for convex feasibility problems in real Hilbert spaces. Furthermore, we study Halpern-type iterative schemes for finding common solutions of a convex feasibility problem and common fixed points of an infinite family of quasi-nonexpansive mappings in Hilbert spaces. Our results improve and generalize many known results in the current literature.
\end{abstract}

\section{INTRODUCTION}

In 1994, Censor and Elfving [19] first introduced the split feasibility problem (SFP) in finite-dimensional Hilbert spaces for modelling inverse problems which arise from phase retrievals and in medical image reconstruction [5]. A number of image reconstruction problems can be formulated as SFP; see, for example, $[1,6]$ and the references therein. The SFP can also be applied to study image processing and intensitymodulated radiation therapy; see, for example, [18] and the references therein. Recently, many authors investigated SFP in different areas of pure and applied mathematics, see [2, 3, 7-17, 20-22, 26, 27, 29, 30, 40, 42-44] for more details. On the other hand,

Received June 26, 2013, accepted September 2, 2013.

Communicated by Jen-Chih Yao.

2010 Mathematics Subject Classification: 47H10, 37C25.

Key words and phrases: Halpern iterative scheme, Convex feasibility problem, Split common fixed-point problem, Quasi-nonexpansive mapping, Fixed point, Strong convergence. 
the split common fixed-point problem (SCFP) is a generalization of the split feasibility problem (SFP) and the convex feasibility problem (CFP); see [21]. In this paper we introduce and study the convergence properties of Halpern-type algorithms for solving the SCFP for the class of quasi-nonexpansive operators $\mathrm{T}$ such that $T-I$ is closed at the origin. This general class, which properly includes the class of directed operators considered in [4, 21], is more desirable, for example, in fixed-point methods in image recovery where, in many cases, it is possible to map the set of images possessing a certain property to the fixed point set of a nonlinear quasi-nonexpansive operator.

Throughout this paper, we denote the set of real numbers and the set of positive integers by $\mathbb{R}$ and $\mathbb{N}$, respectively. Let $H$ be a real Hilbert space with the norm $\|$.$\| and the inner product \langle.,$.$\rangle . When \left\{x_{n}\right\}_{n \in \mathbb{N}}$ is a sequence in $H$, we denote the strong convergence of $\left\{x_{n}\right\}_{n \in \mathbb{N}}$ to $x \in H$ by $x_{n} \rightarrow x$ and the weak convergence by $x_{n} \rightarrow x$. Let $C$ be a nonempty subset of $H$ and let $T: C \rightarrow H$ be a mapping. We denote by $F(T)$ the set of fixed points of $T$, i.e., $F(T)=\{x \in C: T x=x\}$. A mapping $T: C \rightarrow H$ is said to be nonexpansive if $\|T x-T y\| \leq\|x-y\|$ for all $x, y \in C$. A mapping $T: C \rightarrow X$ is said to be quasi-nonexpansive if $F(T) \neq \emptyset$ and $\|T x-y\| \leq\|x-y\|$ for all $x \in C$ and $y \in F(T)$. Let $C$ be a nonempty, closed and convex subset of $H$ and $x \in H$. Then there exists a unique nearest point $z \in C$ such that $\|x-z\|=\inf _{y \in C}\|x-y\|$. We denote such correspondence by $z=P_{C} x$. The mapping $P_{C}$ is called metric projection of $H$ onto $C$.

Our main purpose here is to give an extension of the unified framework developed in [31] to quasi-nonexpansive operators by proposing a strong convergence result of the algorithm we will introduce. This will be done in the context of general Hilbert spaces.

Let us begin with the following Moudafi [32] convex feasibility problem:

$$
\text { Find } x \in C, y \in Q \text { such that } A x=B y \text {, }
$$

where $H_{1}, H_{2}, H_{3}$ are real Hilbert spaces, $C \subset H_{1}, Q \subset H_{2}$ are two nonempty, closed and convex level sets, and $A: H_{1} \rightarrow H_{3}, B: H_{2} \rightarrow H_{3}$ are two bounded linear operators.

The split feasibility problem (SFP) [19] is formulated as finding a point $x$ with the property

$$
x \in C \text { such that } A x \in Q \text {, }
$$

where $C$ is a closed and convex subset of a Hilbert space $H_{1}, Q$ is a closed and convex subset of a Hilbert space $H_{2}$, and $A: H_{1} \rightarrow H_{2}$ is a bounded linear operator.

Assuming that the SFP is consistent (i.e., (1.2) has a solution), it is not hard to see that $x \in C$ solves (1.2) if and only if it solves the fixed-point equation

$$
x=P_{C}\left(I-\gamma A^{*}\left(I-P_{Q}\right) A\right) x, \quad x \in C,
$$


where $P_{C}$ and $P_{Q}$ are the (orthogonal) projections onto $C$ and $Q$, respectively, $\gamma>0$ is any positive constant, and $A^{*}$ denotes the adjoint of $A$.

To solve (1.2), Byrne [5] proposed his CQ algorithm, which generates a sequence $\left\{x_{n}\right\}_{n \in \mathbb{N}}$ by

$$
x_{n+1}=P_{C}\left(I-\gamma A^{*}\left(I-P_{Q}\right) A\right) x_{n}, \quad n \in \mathbb{N},
$$

where $\gamma \in\left(0, \frac{2}{\lambda}\right)$, with $\lambda$ being the spectral radius of the operator $A^{*} A$.

Very recently, Moudafi considered in [32] the problem (1.1) and introduced the following alternating CQ-algorithm, namely

$$
\text { (ACQA) }\left\{\begin{array}{l}
x_{0} \in H_{1}, y_{0} \in H_{2} \quad \text { chosen arbitrarily, } \\
x_{n+1}=P_{C}\left(x_{n}-\gamma_{n} A^{*}\left(A x_{n}-B y_{n}\right)\right), \\
y_{n+1}=P_{Q}\left(y_{n}+\gamma_{n} B^{*}\left(A x_{n+1}-B y_{n}\right)\right) .
\end{array}\right.
$$

Then he proved the weak convergence of the sequence $\left\{\left(x_{n}, y_{n}\right)\right\}_{n \in \mathbb{N}}$ to a solution of (1.1) provided that the solution set $\Omega=\{(x, y) \in C \times Q: A x=B y\}$ is nonempty and some conditions on the sequence of positive parameters $\left\{\gamma_{n}\right\}_{n \in \mathbb{N}}$. The ACQA involves two projections $P_{C}$ and $P_{Q}$ and hence might be hard to be implemented in the case where one of them fails to have a closed-form expression. He also introduced the closed and convex sets $C$ and $Q$ as level sets:

$$
C=\left\{x \in H_{1}: c(x) \leq 0\right\} \quad \text { and } \quad Q=\left\{y \in H_{2}: q(y) \leq 0\right\},
$$

where $c: H_{1} \rightarrow \mathbb{R}$ and $q: H_{2} \rightarrow \mathbb{R}$ are two convex functions which are subdifferentiable on $C$ and $Q$ respectively and their subdifferentials are bounded on bounded sets and finally considered [32] the following relaxed alternating CQ-algorithm

$$
(\text { RACQA })\left\{\begin{array}{l}
x_{0} \in H_{1}, y_{0} \in H_{2} \quad \text { chosen arbitrarily, } \\
x_{n+1}=P_{C_{n}}\left(x_{n}-\gamma A^{*}\left(A x_{n}-B y_{n}\right)\right), \\
y_{n+1}=P_{Q_{n}}\left(y_{n}+\gamma B^{*}\left(A x_{n+1}-B y_{n}\right)\right),
\end{array}\right.
$$

where $\gamma>0$ and

$$
C_{n}=\left\{x \in H_{1}: c\left(x_{n}\right)+\left\langle\xi_{n}, x-x_{n}\right\rangle \leq 0\right\}, \quad \xi_{n} \in \partial c\left(x_{n}\right)
$$

and

$$
Q_{n}=\left\{y \in H_{2}: q\left(y_{n}\right)+\left\langle\eta_{n}, y-y_{n}\right\rangle \leq 0\right\}, \quad \eta_{n} \in \partial q\left(y_{n}\right) .
$$

It is obvious that $C \subset C_{n}$ and $Q \subset Q_{n}$ for all $n \in \mathbb{N}$ and $C_{n}$ and $Q_{n}$ are half-spaces. Thus the corresponding projections have closed-form expressions.

Moudafi [32] studied convex feasibility problems and proved the following weak convergence theorem in Hilbert spaces. 
Theorem 1.1. Let $H_{1}, H_{2}$ and $H_{3}$ be real Hilbert spaces, $C \subset H_{1}, Q \subset H_{2}$ be two nonempty, closed and convex sets, $A: H_{1} \rightarrow H_{3}, B: H_{2} \rightarrow H_{3}$ be two bounded linear operators. Assume that $\Omega$ is the solution set of (1.1) and that $\gamma \in$ $\left(0, \min \left\{\frac{1}{\|A\|^{2}}, \frac{1}{\|B\|^{2}}\right\}\right)$. Let $\left\{x_{n}\right\}_{n \in \mathbb{N}}$ be a sequence generated by algorithm (1.6). If $\Omega \neq \varnothing$, then the sequence $\left\{\left(x_{n}, y_{n}\right)\right\}_{n \in \mathbb{N}}$ converges weakly to a solution $(\bar{x}, \bar{y})$ of (1.1).

The following open question was raised by Moudafi in his final remark of [32].

Question 1.1. Is there any strong convergence theorem of a relaxed alternating CQ-algorithm (RACQA) for convex feasibility problem (1.1) in real Hilbert spaces?

The concept of nonexpansivity plays an important role in the study of Halpern-type iteration for finding fixed points of a mapping $T: C \rightarrow C$, where $C$ is a nonempty, closed and convex subset of a real Banach space. Recall that the one-step Halpern iteration is given by the following formula

$$
x_{n+1}=\alpha_{n} u+\left(1-\alpha_{n}\right) T x_{n}, \quad u \in C, x_{1} \in C .
$$

Here, $\left\{\alpha_{n}\right\}_{n \in \mathbb{N}}$ is a real sequence in $[0,1]$ satisfying some appropriate conditions. A more general iteration scheme of one-step Halpern iteration is two-step Halpern iteration given by

$$
\left\{\begin{array}{l}
u \in C, x_{1} \in C \quad \text { chosen arbitrarily, } \\
y_{n}=\left(1-\beta_{n}\right) x_{n}+\beta_{n} T x_{n} \\
x_{n+1}=\alpha_{n} u+\left(1-\alpha_{n}\right) y_{n}
\end{array}\right.
$$

where the sequences $\left\{\beta_{n}\right\}_{n \in \mathbb{N}}$ and $\left\{\alpha_{n}\right\}_{n \in \mathbb{N}}$ satisfy some appropriate conditions. In particular, when all $\beta_{n}=1$, the Halpern iteration (1.8) becomes the standard Halpern iteration (1.7). The construction of fixed points of nonexpansive mappings via Halpern's algorithm [25] has been extensively investigated recently in the current literature (see, for example, [34] and the references therein). Numerous results have been proved on Halpern's iterations for nonexpansive mappings in Hilbert and Banach spaces (see, e.g., [25, 34, 39, 35, 38, 36, 33]).

In this paper, we introduce a new Halpern-type iterative algorithm for finding a solution of (1.1) and obtain a strongly convergent iterative sequence to a solution of (1.1) in real Hilbert spaces. Consequently, the above question is answered in the affirmative in Hilbert space setting. Our results improve and generalize many known results in the current literature; see, for example, [31, 32].

\section{Preliminaries}

In this section, we collect some lemmas which will be used in the proofs of the main results in next sections. We start with the following well known lemma. 
Lemma 2.1. ([37]). Let $H$ be a real Hilbert space and and $C$ a nonempty, closed and convex subset of $H$. For given $x \in H$ :

(i) $z=P_{C} x$ if and only if

$$
\langle x-z, y-z\rangle \leq 0, \quad \forall y \in C .
$$

(ii) $z=P_{C} x$ if and only if

$$
\|x-z\|^{2} \leq\|x-y\|^{2}-\|y-z\|^{2}, \quad \forall y \in C .
$$

(iii) $\left\langle P_{C} x-P_{C} y, x-y\right\rangle \geq\left\|P_{C} x-P_{C} y\right\|^{2}, \forall x, y \in H$. Consequently, $P_{C}$ is a nonexpansive mapping.

Lemma 2.2. ([24]). In a Hilbert space $H$, we have

(i) for all $x, y \in H$ and $\lambda \in[0,1]$

$$
\|\lambda x+(1-\lambda) y\|^{2}=\lambda\|x\|^{2}+(1-\lambda)\|y\|^{2}-\lambda(1-\lambda)\|x-y\|^{2} .
$$

(ii) for all $x, y, z \in H$

$$
\|x-y\|^{2} \leq\|x-(y+z)\|^{2}+2\langle x-y, z\rangle .
$$

(iii) for all $x, y \in H$

$$
\|x+y\|^{2} \leq\|x\|^{2}+2\langle y, x+y\rangle .
$$

Lemma 2.3. ([32]). Let $T$ be a quasi-nonexpansive mapping of a Hilbert space $H$, and set $T_{\alpha}=(1-\alpha) I+\alpha T$ for $\alpha \in(0,1]$. Then, the following are reached for all $(x, q) \in H \times F(T)$ :

(i) $\langle x-T x, x-q\rangle \geq \frac{1}{2}\|x-T x\|^{2}$ and $\langle x-T x, q-T x\rangle \leq \frac{1}{2}\|x-T x\|^{2}$;

(ii) $\left\|T_{\alpha} x-q\right\|^{2} \leq\|x-q\|^{2}-\alpha(1-\alpha)\|T x-x\|^{2}$;

(iii) $\left\langle x-T_{\alpha} x, x-q\right\rangle \geq \frac{\alpha}{2}\|x-T x\|^{2}$.

Definition 2.1. ([32]). A mapping $T$ is said to be demiclosed if, for any sequence $\left\{x_{n}\right\}_{n \in \mathbb{N}}$ which weakly converges to $y$, and if the sequence $\left\{T x_{n}\right\}_{n \in \mathbb{N}}$ strongly converges to $z$, then $T y=z$.

If $X$ and $Y$ are inner product spaces, let $X \times Y$ denote the set of all ordered pairs $(x, y)$ with $x \in X$ and $y \in Y$. Addition and scalar multiplication are defined "componentwise" in $X \times Y$; that is,

$$
\left(x_{1}, y_{1}\right)+\left(x_{2}, y_{2}\right):=\left(x_{1}+x_{2}, y_{1}+y_{2}\right),
$$

and

$$
\alpha(x, y):=(\alpha x, \alpha y) .
$$

Now, we define an inner product in $X \times Y$ by

$$
\left\langle\left(x_{1}, y_{1}\right),\left(x_{2}, y_{2}\right)\right\rangle:=\left\langle x_{1}, x_{2}\right\rangle+\left\langle y_{1}, y_{2}\right\rangle
$$


The norm in $X \times Y$ is given by

$$
\|(x, y)\|:=\sqrt{\|x\|^{2}+\|y\|^{2}} .
$$

In particular, convergence in $X \times Y$ is componentwise:

$$
\left\|\left(x_{n}, y_{n}\right)-(x, y)\right\| \rightarrow 0 \Longleftrightarrow\left\|x_{n}-x\right\| \rightarrow 0 \text { and }\left\|y_{n}-y\right\| \rightarrow 0
$$

Proposition 2.1. ([23]). Let $X$ and $Y$ be two inner product spaces. Then $X \times Y$ is an inner product space. It is complete if both $X$ and $Y$ are complete.

To prove our main result, we need the following key lemmas.

Lemma 2.4. ([41]). Let $\left\{s_{n}\right\}_{n \in \mathbb{N}}$ be a sequence of nonnegative real numbers satisfying the inequality:

$$
s_{n+1} \leq\left(1-\gamma_{n}\right) s_{n}+\gamma_{n} \delta_{n}, \quad \forall n \geq 0,
$$

where $\left\{\gamma_{n}\right\}_{n \in \mathbb{N}}$ and $\left\{\delta_{n}\right\}_{n \in \mathbb{N}}$ satisfy the conditions:

(i) $\left\{\gamma_{n}\right\}_{n \in \mathbb{N}} \subset[0,1]$ and $\sum_{n=0}^{\infty} \gamma_{n}=\infty$, or equivalently, $\Pi_{n=0}^{\infty}\left(1-\gamma_{n}\right)=0$;

(ii) $\lim \sup _{n \rightarrow \infty} \delta_{n} \leq 0$, or

(ii') $\sum_{n=0}^{\infty} \gamma_{n} \delta_{n}<\infty$.

Then, $\lim _{n \rightarrow \infty} s_{n}=0$.

Lemma 2.5. ([28]). Let $\left\{a_{n}\right\}_{n \in \mathbb{N}}$ be a sequence of real numbers such that there exists a subsequence $\left\{n_{i}\right\}_{i \in \mathbb{N}}$ of $\{n\}_{n \in \mathbb{N}}$ such that $a_{n_{i}}<a_{n_{i}+1}$ for all $i \in \mathbb{N}$. Then there exists a subsequence $\left\{m_{k}\right\}_{k \in \mathbb{N}} \subset \mathbb{N}$ such that $m_{k} \rightarrow \infty$ and the following properties are satisfied by all (sufficiently large) numbers $k \in \mathbb{N}$ :

$$
a_{m_{k}} \leq a_{m_{k}+1} \quad \text { and } \quad a_{k} \leq a_{m_{k}+1} .
$$

In fact, $m_{k}=\max \left\{j \leq k: a_{j}<a_{j+1}\right\}$.

Let $E$ be a real Banach space. The modulus $\delta$ of convexity of $E$ is denoted by

$$
\delta(\epsilon)=\inf \left\{1-\frac{\|x+y\|}{2}:\|x\| \leq 1,\|y\| \leq 1,\|x-y\| \geq \epsilon\right\}
$$

for every $\epsilon$ with $0 \leq \epsilon \leq 2$. A Banach space $X$ is said to be uniformly convex if $\delta(\epsilon)>0$ for every $\epsilon>0$. It is well known that any Hilbert space is a uniformly convex Banach space; see [37], for more details.

Lemma 2.6. ([22]). Let $E$ be a uniformly convex Banach space, $r>0$ be a constant. Then there exists a continuous, strictly increasing and convex function $h:[0, \infty) \rightarrow[0, \infty)$ such that

$$
\left\|\sum_{k=0}^{\infty} \alpha_{k} x_{k}\right\|^{2} \leq \sum_{k=0}^{\infty} \alpha_{k}\left\|x_{k}\right\|^{2}-\alpha_{i} \alpha_{j} h\left(\left\|x_{i}-x_{j}\right\|\right)
$$


for all $i, j \in \mathbb{N} \cup\{0\}, x_{k} \in B_{r}:=\{z \in E:\|z\| \leq r\}, \alpha_{k} \in(0,1)$ and $k \in \mathbb{N} \cup\{0\}$ with $\sum_{k=0}^{\infty} \alpha_{k}=1$.

\section{Strong Convergence Theorems for Convex Feasibility Problems}

In this section, we study convex feasibility problem (1.1) and prove the following two strong convergence theorems in Hilbert spaces.

Theorem 3.1. Let $H_{1}, H_{2}$ and $H_{3}$ be real Hilbert spaces, $C \subset H_{1}, Q \subset H_{2}$ be two nonempty, closed and convex sets as level sets:

$$
C=\left\{x \in H_{1}: f(x) \leq 0\right\} \quad \text { and } \quad Q=\left\{y \in H_{2}: g(y) \leq 0\right\},
$$

where $f: H_{1} \rightarrow \mathbb{R}$ and $g: H_{2} \rightarrow \mathbb{R}$ are two convex functions which are subdifferentiable on $C$ and $Q$ respectively and their subdifferentials are bounded on bounded sets. Let $A: H_{1} \rightarrow H_{3}, B: H_{2} \rightarrow H_{3}$ be two bounded linear operators. Assume that $\gamma \in\left(0, \min \left\{\frac{1}{\|A\|^{2}}, \frac{1}{\|B\|^{2}}\right\}\right)$. Let $(u, w) \in H_{1} \times H_{2}$ be fixed. Let $\left\{\alpha_{n}\right\}_{n \in \mathbb{N}}$ be a sequence in $[0,1]$ satisfying the following control conditions:

(a) $\lim _{n \rightarrow \infty} \alpha_{n}=0$;

(b) $\sum_{n=1}^{\infty} \alpha_{n}=\infty$.

Let $\left\{x_{n}\right\}_{n \in \mathbb{N}}$ be a sequence generated by

$$
\left\{\begin{array}{l}
x_{0} \in H_{1}, y_{0} \in H_{2} \quad \text { chosen arbitrarily, } \\
u_{n}=P_{C_{n}}\left(x_{n}-\gamma A^{*}\left(A x_{n}-B y_{n}\right)\right) \\
w_{n}=P_{Q_{n}}\left(y_{n}+\gamma B^{*}\left(A x_{n+1}-B y_{n}\right)\right) \\
x_{n+1}=\alpha_{n} u+\left(1-\alpha_{n}\right) u_{n} \\
y_{n+1}=\alpha_{n} w+\left(1-\alpha_{n}\right) w_{n} .
\end{array}\right.
$$

where $C_{n}=\left\{x \in H_{1}: f\left(x_{n}\right)+\left\langle\xi_{n}, x-x_{n}\right\rangle \leq 0\right\}, \xi_{n} \in \partial f\left(x_{n}\right)$ and $Q_{n}=\left\{y \in H_{2}:\right.$ $\left.g\left(y_{n}\right)+\left\langle\eta_{n}, y-y_{n}\right\rangle \leq 0\right\}, \eta_{n} \in \partial g\left(y_{n}\right)$. If $\Omega:=\{(x, y) \in C \times Q: A x=B y\} \neq \varnothing$, then the sequence $\left\{\left(x_{n}, y_{n}\right)\right\}_{n \in \mathbb{N}}$ defined in (3.1) converges strongly to $P_{\Omega}(u, w)$, where $P_{\Omega}$ is the metric projection from $H_{1} \times H_{2}$ onto $\Omega$.

Proof. We divide the proof into several steps. We first note that, in view of Proposition 2.1, $H_{1} \times H_{2}$ is a real Hilbert space. It could easily be seen that $C \subset C_{n}$ and $Q \subset Q_{n}$ for all $n \in \mathbb{N}$ and $C_{n}$ and $Q_{n}$ are half-spaces. It is also clear that $\Omega$ is a closed and convex subset of $C \times Q$. Set

$$
(\hat{x}, \hat{y})=P_{\Omega}(u, w) .
$$

Step 1. We prove that the sequences $\left\{x_{n}\right\}_{n \in \mathbb{N}},\left\{y_{n}\right\}_{n \in \mathbb{N}},\left\{u_{n}\right\}_{n \in \mathbb{N}},\left\{w_{n}\right\}_{n \in \mathbb{N}}$, $\left\{A x_{n}\right\}_{n \in \mathbb{N}}$ and $\left\{B y_{n}\right\}_{n \in \mathbb{N}}$ are bounded. We first show that $\left\{x_{n}\right\}_{n \in \mathbb{N}}$ is bounded. Let 
$\left(x^{*}, y^{*}\right) \in \Omega$ be arbitrarily chosen. Then $x^{*} \in C$ (and thus $x^{*} \in C_{n}$ ), $y^{*} \in Q$ (and thus $y^{*} \in Q_{n}$ ) and $A x^{*}=B y^{*}$. In view of Lemma 2.1(iii) and (3.1), we have

$$
\begin{aligned}
& \left\|u_{n}-x^{*}\right\|^{2} \\
= & \left\|P_{C_{n}}\left(x_{n}-\gamma A^{*}\left(A x_{n}-B y_{n}\right)\right)-P_{C_{n}} x^{*}\right\|^{2} \\
\leq & \left\|x_{n}-\gamma A^{*}\left(A x_{n}-B y_{n}\right)-x^{*}\right\|^{2} \\
= & \left\|x_{n}-x^{*}\right\|^{2}+\gamma^{2}\left\|A^{*}\left(A x_{n}-B y_{n}\right)\right\|^{2}-2 \gamma\left\langle A^{*}\left(A x_{n}-B y_{n}\right), x_{n}-x^{*}\right\rangle \\
\leq & \left\|x_{n}-x^{*}\right\|^{2}+\gamma^{2}\|A\|^{2}\left\|A x_{n}-B y_{n}\right\|^{2}-2 \gamma\left\langle A x_{n}-B y_{n}, A x_{n}-A x^{*}\right\rangle .
\end{aligned}
$$

Let $\theta_{n}:=2 \gamma\left\langle A x_{n}-B y_{n}, A x_{n}-A x^{*}\right\rangle$. Then we obtain

$$
\theta_{n}=2 \gamma\left(\left\|A x_{n}-B y_{n}\right\|^{2}+\left\langle A x_{n}-B y_{n}, B y_{n}-A x^{*}\right\rangle\right) .
$$

This implies that

$$
\begin{aligned}
& \left\|u_{n}-x^{*}\right\|^{2} \\
\leq & \left\|x_{n}-x^{*}\right\|^{2}-2 \gamma\left\langle A x_{n}-B y_{n}, B y_{n}-A x^{*}\right\rangle-\gamma\left(2-\gamma\|A\|^{2}\right)\left\|A x_{n}-B y_{n}\right\|^{2} .
\end{aligned}
$$

Similarly, we have

$$
\begin{gathered}
\left\|w_{n}-y^{*}\right\|^{2} \leq\left\|y_{n}-y^{*}\right\|^{2}+2 \gamma\left\langle A x_{n+1}-B y_{n}, A x_{n+1}\right. \\
\left.-B y^{*}\right\rangle-\gamma\left(2-\gamma\|B\|^{2}\right)\left\|A x_{n+1}-B y_{n}\right\|^{2} .
\end{gathered}
$$

On the other hand, we have

$$
\begin{aligned}
& 2\left\langle A x_{n}-B y_{n}, B y_{n}-A x^{*}\right\rangle \\
= & -\left\|A x_{n}-B y_{n}\right\|^{2}-\left\|B y_{n}-A x^{*}\right\|^{2}+\left\|A x_{n}-B y^{*}\right\|^{2} \\
= & -\left\|A x_{n}-B y_{n}\right\|^{2}-\left\|B y_{n}-B y^{*}\right\|^{2}+\left\|A x_{n}-A x^{*}\right\|^{2}
\end{aligned}
$$

and

$$
\begin{aligned}
& 2\left\langle B y_{n}-A x_{n+1}, A x_{n+1}-B y^{*}\right\rangle \\
= & -\left\|B y_{n}-A x_{n+1}\right\|^{2}-\left\|A x_{n+1}-B y^{*}\right\|^{2}+\left\|B y_{n}-A x^{*}\right\|^{2} \\
= & -\left\|B y_{n}-A x_{n+1}\right\|^{2}-\left\|A x_{n+1}-A x^{*}\right\|^{2}+\left\|B y_{n}-B y^{*}\right\|^{2}
\end{aligned}
$$

It follows from (3.2)-(3.7) that

$$
\begin{aligned}
\left\|u_{n}-x^{*}\right\|^{2} \leq & \left\|x_{n}-x^{*}\right\|^{2}+\gamma\left\|A x_{n}-B y_{n}\right\|^{2}+\gamma\left\|B y_{n}-B y^{*}\right\|^{2} \\
& -\gamma\left\|A x_{n}-A x^{*}\right\|^{2}-\gamma\left(2-\gamma\|A\|^{2}\right)\left\|A x_{n}-B y_{n}\right\|^{2}
\end{aligned}
$$

and

$$
\begin{aligned}
\left\|w_{n}-y^{*}\right\|^{2} \leq & \left\|y_{n}-y^{*}\right\|^{2}+\gamma\left\|A x_{n+1}-B y_{n}\right\|^{2}+\gamma\left\|A x_{n+1}-A x^{*}\right\|^{2} \\
& -\gamma\left\|B y_{n}-B y^{*}\right\|^{2}-\gamma\left(2-\gamma\|B\|^{2}\right)\left\|A x_{n+1}-B y_{n}\right\|^{2}
\end{aligned}
$$


Adding these two inequalities, we conclude that

$$
\begin{aligned}
& \left\|u_{n}-x^{*}\right\|^{2}+\left\|w_{n}-y^{*}\right\|^{2} \\
\leq & \left\|x_{n}-x^{*}\right\|^{2}+\left\|y_{n}-y^{*}\right\|^{2}-\gamma\left\|A x_{n}-A x^{*}\right\|^{2}+\gamma\left\|A x_{n+1}-A x^{*}\right\|^{2} \\
& -\gamma\left(1-\gamma\|A\|^{2}\right)\left\|A x_{n}-B y_{n}\right\|^{2}-\gamma\left(1-\gamma\|B\|^{2}\right)\left\|A x_{n+1}-B y_{n}\right\|^{2}
\end{aligned}
$$

In view of (3.1) and (3.10), we obtain

$$
\begin{aligned}
& \left\|x_{n+1}-x^{*}\right\|^{2}+\left\|y_{n+1}-y^{*}\right\|^{2} \\
= & \left\|\alpha_{n} u+\left(1-\alpha_{n}\right) u_{n}-x^{*}\right\|^{2} \\
& +\left\|\alpha_{n} w+\left(1-\alpha_{n}\right) w_{n}-y^{*}\right\|^{2} \\
\leq & \alpha_{n}\left\|u-x^{*}\right\|^{2}+\left(1-\alpha_{n}\right)\left\|u_{n}-x^{*}\right\|^{2} \\
& +\alpha_{n}\left\|w-y^{*}\right\|^{2}+\left(1-\alpha_{n}\right)\left\|w_{n}-y^{*}\right\|^{2} \\
= & \alpha_{n}\left[\left\|u-x^{*}\right\|^{2}+\left\|w-y^{*}\right\|^{2}\right]+\left(1-\alpha_{n}\right)\left[\left\|u_{n}-x^{*}\right\|^{2}+\left\|w_{n}-y^{*}\right\|^{2}\right] \\
\leq & \alpha_{n}\left[\left\|u-x^{*}\right\|^{2}+\left\|w-y^{*}\right\|^{2}\right]+\left(1-\alpha_{n}\right)\left[\left\|x_{n}-x^{*}\right\|^{2}+\left\|y_{n}-y^{*}\right\|^{2}\right. \\
& -\gamma\left\|A x_{n}-A x^{*}\right\|^{2}+\gamma\left\|A x_{n+1}-A x^{*}\right\|^{2} \\
& \left.-\gamma\left(1-\gamma\|A\|^{2}\right)\left\|A x_{n}-B y_{n}\right\|^{2}-\gamma\left(1-\gamma\|B\|^{2}\right)\left\|A x_{n+1}-B y_{n}\right\|^{2}\right] \\
\leq & \alpha_{n}\left[\left\|u-x^{*}\right\|^{2}+\left\|w-y^{*}\right\|^{2}\right]+\gamma\left\|A x_{n+1}-A x^{*}\right\|^{2} \\
& +\left(1-\alpha_{n}\right)\left[\left\|x_{n}-x^{*}\right\|^{2}+\left\|y_{n}-y^{*}\right\|^{2}\right. \\
& \left.-\gamma\left\|A x_{n}-A x^{*}\right\|^{2}\right]-\left(1-\alpha_{n}\right)\left[\gamma\left(1-\gamma\|A\|^{2}\right)\left\|A x_{n}-B y_{n}\right\|^{2}\right. \\
& \left.+\gamma\left(1-\gamma\|B\|^{2}\right)\left\|A x_{n+1}-B y_{n}\right\|^{2}\right]
\end{aligned}
$$

Put

$$
E_{n}\left(x^{*}, y^{*}\right):=\left\|x_{n}-x^{*}\right\|^{2}+\left\|y_{n}-y^{*}\right\|^{2}-\gamma\left\|A x_{n}-A x^{*}\right\|^{2} .
$$

We note that

$$
\gamma\left\|A x_{n}-A x^{*}\right\|^{2} \leq \gamma\|A\|^{2}\left\|x_{n}-x^{*}\right\|^{2} .
$$

Therefore

$$
E_{n}\left(x^{*}, y^{*}\right) \geq\left(1-\gamma\|A\|^{2}\right)\left\|x_{n}-x^{*}\right\|^{2}+\left\|y_{n}-y^{*}\right\|^{2} \geq 0 .
$$

In view of (3.11)-(3.13), we conclude that

$$
\begin{aligned}
E_{n+1}\left(x^{*}, y^{*}\right) \leq & \left(1-\alpha_{n}\right) E_{n}\left(x^{*}, y^{*}\right)+\alpha_{n}\left[\left\|u-x^{*}\right\|^{2}+\left\|w-y^{*}\right\|^{2}\right] \\
& -\left(1-\alpha_{n}\right)\left[\gamma\left(1-\gamma\|A\|^{2}\right)\left\|A x_{n}-B y_{n}\right\|^{2}\right. \\
& \left.+\gamma\left(1-\gamma\|B\|^{2}\right)\left\|A x_{n+1}-B y_{n}\right\|^{2}\right] .
\end{aligned}
$$


This implies that

$$
\begin{aligned}
E_{n+1}\left(x^{*}, y^{*}\right) & \leq\left(1-\alpha_{n}\right) E_{n}\left(x^{*}, y^{*}\right)+\alpha_{n}\left[\left\|u-x^{*}\right\|^{2}+\left\|w-y^{*}\right\|^{2}\right] \\
& \leq \max \left\{E_{n}\left(x^{*}, y^{*}\right),\left\|u-x^{*}\right\|^{2}+\left\|w-y^{*}\right\|^{2}\right\}
\end{aligned}
$$

By induction, we obtain

$$
E_{n+1}\left(x^{*}, y^{*}\right) \leq \max \left\{E_{1}\left(x^{*}, y^{*}\right),\left\|u-x^{*}\right\|^{2}+\left\|w-y^{*}\right\|^{2}\right\}
$$

for all $n \in \mathbb{N}$. This implies that the sequence $\left\{E_{n}\left(x^{*}, y^{*}\right)\right\}_{n \in \mathbb{N}}$ is bounded and hence, in view of (3.13), the sequences $\left\{x_{n}\right\}_{n \in \mathbb{N}}$ and $\left\{y_{n}\right\}_{n \in \mathbb{N}}$ are bounded. This, together with (3.1), implies that the sequences $\left\{u_{n}\right\}_{n \in \mathbb{N}},\left\{w_{n}\right\}_{n \in \mathbb{N}},\left\{A x_{n}\right\}_{n \in \mathbb{N}}$ and $\left\{B y_{n}\right\}_{n \in \mathbb{N}}$ are bounded too.

In view of (3.14), we conclude that

$$
\begin{aligned}
E_{n+1}(\hat{x}, \hat{y}) \leq & \left(1-\alpha_{n}\right) E_{n}(\hat{x}, \hat{y})+\alpha_{n}\left[\|u-\hat{x}\|^{2}+\|w-\hat{y}\|^{2}\right. \\
& +\gamma\left(1-\gamma\|A\|^{2}\right)\left\|A x_{n}-B y_{n}\right\|^{2} \\
& \left.+\gamma\left(1-\gamma\|B\|^{2}\right)\left\|A x_{n+1}-B y_{n}\right\|^{2}\right] \\
& -\left[\gamma\left(1-\gamma\|A\|^{2}\right)\left\|A x_{n}-B y_{n}\right\|^{2}\right. \\
& \left.+\gamma\left(1-\gamma\|B\|^{2}\right)\left\|A x_{n+1}-B y_{n}\right\|^{2}\right] .
\end{aligned}
$$

Let

$$
\begin{aligned}
M_{1}:= & \sup \left\{\|u-\hat{x}\|^{2}+\|w-\hat{y}\|^{2}+\gamma\left(1-\gamma\|A\|^{2}\right)\left\|A x_{n}-B y_{n}\right\|^{2}\right. \\
& \left.+\gamma\left(1-\gamma\|B\|^{2}\right)\left\|A x_{n+1}-B y_{n}\right\|^{2}: n \in \mathbb{N}\right\} .
\end{aligned}
$$

It follows from (3.15) that

$$
\begin{aligned}
& \gamma\left(1-\gamma\|A\|^{2}\right)\left\|A x_{n}-B y_{n}\right\|^{2}+\gamma\left(1-\gamma\|B\|^{2}\right)\left\|A x_{n+1}-B y_{n}\right\|^{2} \\
\leq & E_{n}(\hat{x}, \hat{y})-E_{n+1}(\hat{x}, \hat{y})+\alpha_{n} M_{1} .
\end{aligned}
$$

Step 2. We prove that for any $n \in \mathbb{N}$

$$
\begin{aligned}
E_{n+1}(\hat{x}, \hat{y}) \leq & \left(1-\alpha_{n}\right) E_{n}(\hat{x}, \hat{y}) \\
& +\alpha_{n}\left[\gamma\left(1-\gamma\|A\|^{2}\right)\left\|A x_{n}-B y_{n}\right\|^{2}\right. \\
& +\gamma\left(1-\gamma\|B\|^{2}\right)\left\|A x_{n+1}-B y_{n}\right\|^{2} \\
& \left.+2\left\langle\left(x_{n+1}, y_{n+1}\right)-(\hat{x}, \hat{y}),(u, w)-(\hat{x}, \hat{y})\right\rangle\right] .
\end{aligned}
$$

Let us show (3.17). In view of Lemma 2.2(iii), we obtain 


$$
\begin{aligned}
& E_{n+1}(\hat{x}, \hat{y}) \\
= & \left\|x_{n+1}-\hat{x}\right\|^{2}+\left\|y_{n+1}-\hat{y}\right\|^{2}-\gamma\left\|A x_{n+1}-A \hat{x}\right\|^{2} \\
= & \left\|\left(x_{n+1}, y_{n+1}\right)-(\hat{x}, \hat{y})\right\|^{2}-\gamma\left\|A x_{n+1}-A \hat{x}\right\|^{2} \\
= & \left\|\alpha_{n}(u, w)+\left(1-\alpha_{n}\right)\left(u_{n}, w_{n}\right)-(\hat{x}, \hat{y})\right\|^{2}-\gamma\left\|A x_{n+1}-A \hat{x}\right\|^{2} \\
\leq & \left(1-\alpha_{n}\right)\left\|\left(u_{n}, w_{n}\right)-(\hat{x}, \hat{y})\right\|^{2} \\
& +2 \alpha_{n}\left\langle\left(x_{n+1}, y_{n+1}\right)-(\hat{x}, \hat{y}),(u, w)-(\hat{x}, \hat{y})\right\rangle-\gamma\left\|A x_{n+1}-A \hat{x}\right\|^{2} \\
\leq & \left(1-\alpha_{n}\right)\left[\left\|u_{n}-\hat{x}\right\|^{2}+\left\|w_{n}-\hat{y}\right\|^{2}\right] \\
& +2 \alpha_{n}\left\langle\left(x_{n+1}, y_{n+1}\right)-(\hat{x}, \hat{y}),(u, w)-(\hat{x}, \hat{y})\right\rangle-\gamma\left\|A x_{n+1}-A \hat{x}\right\|^{2} \\
\leq & \left(1-\alpha_{n}\right)\left[\left\|x_{n}-\hat{x}\right\|^{2}+\left\|y_{n}-\hat{y}\right\|^{2}-\gamma\left\|A x_{n}-A \hat{x}\right\|^{2}+\gamma\left\|A x_{n+1}-A \hat{x}\right\|^{2}\right. \\
& \left.-\gamma\left(1-\gamma\|A\|^{2}\right)\left\|A x_{n}-B y_{n}\right\|^{2}-\gamma\left(1-\gamma\|B\|^{2}\right)\left\|A x_{n+1}-B y_{n}\right\|^{2}\right] \\
& +2 \alpha_{n}\left\langle\left(x_{n+1}, y_{n+1}\right)-(\hat{x}, \hat{y}),(u, w)-(\hat{x}, \hat{y})\right\rangle-\gamma\left\|A x_{n+1}-A \hat{x}\right\|^{2} \\
\leq & \left(1-\alpha_{n}\right)\left[\left\|x_{n}-\hat{x}\right\|^{2}+\left\|y_{n}-\hat{y}\right\|^{2}-\gamma\left\|A x_{n}-A \hat{x}\right\|^{2}\right. \\
& \left.-\gamma\left(1-\gamma\|A\|^{2}\right)\left\|A x_{n}-B y_{n}\right\|^{2}-\gamma\left(1-\gamma\|B\|^{2}\right)\left\|A x_{n+1}-B y_{n}\right\|^{2}\right] \\
& +\gamma\left\|A x_{n+1}-A \hat{x}\right\|^{2} \\
& +2 \alpha_{n}\left\langle\left(x_{n+1}, y_{n+1}\right)-(\hat{x}, \hat{y}),(u, w)-(\hat{x}, \hat{y})\right\rangle-\gamma\left\|A x_{n+1}-A \hat{x}\right\|^{2} \\
= & \left(1-\alpha_{n}\right) E_{n}(\hat{x}, \hat{y}) \\
& -\left(1-\alpha_{n}\right)\left[\gamma\left(1-\gamma\|A\|^{2}\right)\left\|A x_{n}-B y_{n}\right\|^{2}+\gamma\left(1-\gamma\|B\|^{2}\right)\left\|A x_{n+1}-B y_{n}\right\|^{2}\right] \\
& +2 \alpha_{n}\left\langle\left(x_{n+1}, y_{n+1}\right)-(\hat{x}, \hat{y}),(u, w)-(\hat{x}, \hat{y})\right\rangle .
\end{aligned}
$$

This implies that

$$
\begin{aligned}
E_{n+1}(\hat{x}, \hat{y}) \leq & \left(1-\alpha_{n}\right) E_{n}(\hat{x}, \hat{y})-\left(1-\alpha_{n}\right)\left[\gamma\left(1-\gamma\|A\|^{2}\right)\left\|A x_{n}-B y_{n}\right\|^{2}\right. \\
& \left.+\gamma\left(1-\gamma\|B\|^{2}\right)\left\|A x_{n+1}-B y_{n}\right\|^{2}\right] \\
& +2 \alpha_{n}\left\langle\left(x_{n+1}, y_{n+1}\right)-(\hat{x}, \hat{y}),(u, w)-(\hat{x}, \hat{y})\right\rangle \\
\leq & \left(1-\alpha_{n}\right) E_{n}(\hat{x}, \hat{y}) \\
& +\alpha_{n}\left[\gamma\left(1-\gamma\|A\|^{2}\right)\left\|A x_{n}-B y_{n}\right\|^{2}+\gamma\left(1-\gamma\|B\|^{2}\right)\left\|A x_{n+1}-B y_{n}\right\|^{2}\right. \\
& \left.+2\left\langle\left(x_{n+1}, y_{n+1}\right)-(\hat{x}, \hat{y}),(u, w)-(\hat{x}, \hat{y})\right\rangle\right] .
\end{aligned}
$$

Thus we have (3.17).

Step 3. We prove that $\left(x_{n}, y_{n}\right) \rightarrow(\hat{x}, \hat{y})$ as $n \rightarrow \infty$.

We shall show that $E_{n}(\hat{x}, \hat{y}) \rightarrow 0$ as $n \rightarrow \infty$ by considering two possible cases on the sequence $\left\{E_{n}(\hat{x}, \hat{y})\right\}_{n \in \mathbb{N}}$.

Case 1. If $\left\{E_{n}(\hat{x}, \hat{y})\right\}_{n \in \mathbb{N}}$ is eventually decreasing, then there exists $n_{0} \in \mathbb{N}$ such that $\left\{E_{n}(\hat{x}, \hat{y})\right\}_{n=n_{0}}^{\infty}$ is decreasing and hence the sequence $\left\{E_{n}(\hat{x}, \hat{y})\right\}_{n \in \mathbb{N}}$ is 
convergent. Thus we have $E_{n}(\hat{x}, \hat{y})-E_{n+1}(\hat{x}, \hat{y}) \rightarrow 0$ as $n \rightarrow \infty$. This, together with (3.16), implies that

(3.18) $\lim _{n \rightarrow \infty}\left[\gamma\left(1-\gamma\|A\|^{2}\right)\left\|A x_{n}-B y_{n}\right\|^{2}+\gamma\left(1-\gamma\|B\|^{2}\right)\left\|A x_{n+1}-B y_{n}\right\|^{2}\right]=0$.

On the other hand, we have

$$
\left\|u_{n}-x_{n}\right\|^{2}=\left\|x_{n}-x^{*}\right\|^{2}-\left\|u_{n}-x^{*}\right\|^{2}+2\left\langle u_{n}-x_{n}, u_{n}-x^{*}\right\rangle .
$$

In view of Lemma 2.1(i) and (3.1), we have

$$
\left\langle x_{n}-\gamma A^{*}\left(A x_{n}-B y_{n}\right)-u_{n}, x^{*}-u_{n}\right\rangle \leq 0 .
$$

This implies that

$\left\langle x_{n}-u_{n}, x^{*}-u_{n}\right\rangle \leq \gamma\left\langle A^{*}\left(A x_{n}-B y_{n}\right), x^{*}-u_{n}\right\rangle \leq \gamma\left\|A^{*}\left(A x_{n}-B y_{n}\right)\right\|\left\|x^{*}-u_{n}\right\|$.

Therefore,

(3.20) $\left\|u_{n}-x_{n}\right\|^{2} \leq\left\|x_{n}-x^{*}\right\|^{2}-\left\|u_{n}-x^{*}\right\|^{2}+2 \gamma\left\|A^{*}\left(A x_{n}-B y_{n}\right)\right\|\left\|x^{*}-u_{n}\right\|$.

In view of (3.1), we deduce that

$$
\lim _{n \rightarrow \infty}\left\|x_{n+1}-u_{n}\right\|=\lim _{n \rightarrow \infty} \alpha_{n}\left\|u-u_{n}\right\|=0 .
$$

Similarly, we obtain

$$
\left\|w_{n}-y_{n}\right\|^{2} \leq\left\|y_{n}-y^{*}\right\|^{2}-\left\|w_{n}-y^{*}\right\|^{2}+2\left\|B^{*}\left(A x_{n+1}-B y_{n}\right)\right\|\left\|y^{*}-w_{n}\right\|
$$

and

$$
\lim _{n \rightarrow \infty}\left\|y_{n+1}-w_{n}\right\|=\lim _{n \rightarrow \infty} \alpha_{n}\left\|w-w_{n}\right\|=0 .
$$

Since $\left\{\left(x_{n}, y_{n}\right)\right\}_{n \in \mathbb{N}}$ is bounded, there exists a subsequence $\left\{\left(x_{n_{i}}, y_{n_{i}}\right)\right\}_{i \in \mathbb{N}}$ of $\left\{\left(x_{n}, y_{n}\right)\right\}_{n \in \mathbb{N}}$ such that $x_{n_{i}} \rightarrow z \in H_{1}, y_{n_{i}} \rightarrow v \in H_{2}, \lim _{i \rightarrow \infty}\left\|x_{n_{i}}-x^{*}\right\|=l\left(x^{*}\right)<\infty$ and

$$
\lim _{i \rightarrow \infty}\left\|y_{n_{i}}-y^{*}\right\|=\lim _{i \rightarrow \infty}\left\|y_{n_{i}+1}-y^{*}\right\|=\lim _{i \rightarrow \infty}\left\|w_{n_{i}}-y^{*}\right\|=l\left(y^{*}\right)<\infty .
$$

This, together with (3.21) and (3.23), implies that $\left\|u_{n_{i}}-x^{*}\right\| \rightarrow l\left(x^{*}\right)$ and $\left\|w_{n_{i}}-y^{*}\right\| \rightarrow$ $l\left(y^{*}\right)$ as $i \rightarrow \infty$ and hence

$$
\lim _{i \rightarrow \infty}\left\|x_{n_{i}}-u_{n_{i}}\right\|=0 \quad \text { and } \quad \lim _{i \rightarrow \infty}\left\|w_{n_{i}}-y_{n_{i}}\right\|=0 .
$$

Since $u_{n_{i}} \in C_{n_{i}}$, we deduce that

$$
f\left(x_{n_{i}}\right) \leq-\left\langle\xi_{n_{i}}, u_{n_{i}}-x_{n_{i}}\right\rangle \leq M_{2}\left\|u_{n_{i}}-x_{n_{i}}\right\|
$$


where $M_{2}$ satisfies $\left\|\xi_{n_{i}}\right\| \leq M_{2}$ for all $i \in \mathbb{N}$. In view of lower semicontinuity of $f$ we conclude that

$$
f(z) \leq \liminf _{i \rightarrow \infty} f\left(x_{n_{i}}\right) \leq 0 .
$$

This implies that $z \in C$. Similarly, since $w_{n_{i}} \in Q_{n_{i}}$, we have

$$
g\left(y_{n_{i}}\right) \leq-\left\langle\eta_{n_{i}}, w_{n_{i}}-y_{n_{i}}\right\rangle \leq M_{3}\left\|w_{n_{i}}-y_{n_{i}}\right\|
$$

where $M_{3}$ satisfies $\left\|\eta_{n_{i}}\right\| \leq M_{3}$ for all $i \in \mathbb{N}$. In view of lower semicontinuity of $g$ we conclude that

$$
g(v) \leq \liminf _{i \rightarrow \infty} g\left(y_{n_{i}}\right) \leq 0 .
$$

This implies that $v \in Q$. By lower semicontiuity of the squared norm and the weak convergence of $\left\{A x_{n_{i}}-B y_{n_{i}}\right\}_{i \in \mathbb{N}}$, we conclude that

$$
\|A z-B v\|^{2} \leq \liminf _{i \rightarrow \infty}\left\|A x_{n_{i}}-B y_{n_{i}}\right\|^{2}=0,
$$

which shows that $(z, v) \in \Omega$. Now, we show the uniqueness of weak cluster points of the sequence $\left\{\left(x_{n}, y_{n}\right)\right\}_{n \in \mathbb{N}}$. To this end, let $\hat{z}, \hat{v}$ be other weak cluster points of $\left\{x_{n}\right\}_{n \in \mathbb{N}}$ and $\left\{y_{n}\right\}_{n \in \mathbb{N}}$, respectively. It follows from (3.12) that

$$
\begin{aligned}
E_{n}(z, v)= & E_{n}(\hat{z}, \hat{v})+\|z-\hat{z}\|^{2}+\|v-\hat{v}\|^{2}-\gamma\|A z-A \hat{z}\|^{2} \\
& +2\left\langle x_{n}-\hat{z}, \hat{z}-z\right\rangle+2\left\langle y_{n}-\hat{v}, \hat{v}-v\right\rangle-2\left\langle A x_{n}-A \hat{z}, A \hat{z}-A z\right\rangle .
\end{aligned}
$$

Letting $n \rightarrow \infty$ we obtain

$$
l(z, v)=l(\hat{z}, \hat{v})+\|z-\hat{z}\|^{2}+\|v-\hat{v}\|^{2}-\gamma\|A z-A \hat{z}\|^{2},
$$

where $l(z, v)=\lim _{n \rightarrow \infty} E_{n}(z, v)$ and $l(\hat{z}, \hat{v})=\lim _{n \rightarrow \infty} E_{n}(\hat{z}, \hat{v})$. Reversing the role of $(z, v)$ and $(\hat{z}, \hat{v})$, we obtain

$$
l(\hat{z}, \hat{v})=l(z, v)+\|z-\hat{z}\|^{2}+\|v-\hat{v}\|^{2}-\gamma\|A z-A \hat{z}\|^{2} .
$$

Since $1-\gamma\|A\|^{2} \geq 0$, we conclude that

$$
\left(1-\gamma\|A\|^{2}\right)\|z-\hat{z}\|^{2}+\|v-\hat{v}\|^{2} \leq 0,
$$

which implies that $(\hat{z}, \hat{v})=(z, v)$. We notice that

$$
\begin{aligned}
& \limsup _{n \rightarrow \infty}\left\langle\left(x_{n}, y_{n}\right)-(\hat{x}, \hat{y}),(u, w)-(\hat{x}, \hat{y})\right\rangle \\
= & \lim _{i \rightarrow \infty}\left\langle\left(x_{n_{i}}, y_{n_{i}}\right)-(\hat{x}, \hat{y}),(u, w)-(\hat{x}, \hat{y})\right\rangle \\
= & \langle(z, v)-(\hat{x}, \hat{y}),(u, w)-(\hat{x}, \hat{y})\rangle .
\end{aligned}
$$


Since $(z, v) \in \Omega$, in view of Lemma 2.1(i), we conclude that

$$
\begin{aligned}
& \limsup _{n \rightarrow \infty}\left\langle\left(x_{n+1}, y_{n+1}\right)-(\hat{x}, \hat{y}),(u, w)-(\hat{x}, \hat{y})\right\rangle \\
\leq & \limsup _{n \rightarrow \infty}\left\langle\left(x_{n+1}, y_{n+1}\right)-\left(x_{n}, y_{n}\right),(u, w)-(\hat{x}, \hat{y})\right\rangle \\
& +\limsup _{n \rightarrow \infty}\left\langle\left(x_{n}, y_{n}\right)-(\hat{x}, \hat{y}),(u, w)-(\hat{x}, \hat{y})\right\rangle \\
= & \limsup _{n \rightarrow \infty}\left\langle\left(x_{n}, y_{n}\right)-(\hat{x}, \hat{y}),(u, w)-(\hat{x}, \hat{y})\right\rangle \\
= & \lim _{i \rightarrow \infty}\left\langle\left(x_{n_{i}}, y_{n_{i}}\right)-(\hat{x}, \hat{y}),(u, w)-(\hat{x}, \hat{y})\right\rangle \\
= & \langle(z, v)-(\hat{x}, \hat{y}),(u, w)-(\hat{x}, \hat{y}) \\
= & \left\langle(z, v)-P_{\Omega}(u, w),(u, w)-P_{\Omega}(u, w)\right\rangle \\
\leq & 0 .
\end{aligned}
$$

Thus we have the desired result by (3.17) and Lemma 2.4.

Case 2. If $\left\{E_{n}(\hat{x}, \hat{y})\right\}_{n \in \mathbb{N}}$ is not eventually decreasing, then there exists a subsequence $\left\{n_{i}\right\}_{i \in \mathbb{N}}$ of $\{n\}_{n \in \mathbb{N}}$ such that

$$
E_{n_{i}}(\hat{x}, \hat{y})<E_{n_{i}+1}(\hat{x}, \hat{y})
$$

for all $i \in \mathbb{N}$. Then, by Lemma 2.5, there exists a nondecreasing sequence $\left\{m_{k}\right\}_{k \in \mathbb{N}} \subset$ $\mathbb{N}$ such that $m_{k} \rightarrow \infty$,

$$
E_{m_{k}}(\hat{x}, \hat{y}) \leq E_{m_{k}+1}(\hat{x}, \hat{y}) \quad \text { and } \quad E_{k}(\hat{x}, \hat{y}) \leq E_{m_{k}+1}(\hat{x}, \hat{y})
$$

for all $k \in \mathbb{N}$. This, together with (3.16), implies that

$$
\begin{aligned}
& \gamma\left(1-\gamma\|A\|^{2}\right)\left\|A x_{m_{k}}-B y_{m_{k}}\right\|^{2}+\gamma\left(1-\gamma\|B\|^{2}\right)\left\|A x_{m_{k}+1}-B y_{m_{k}}\right\|^{2} \\
\leq & E_{m_{k}}(\hat{x}, \hat{y})-E_{m_{k}+1}(\hat{x}, \hat{y})+\alpha_{m_{k}} M_{1} \\
\leq & \alpha_{m_{k}} M_{1} .
\end{aligned}
$$

for all $k \in \mathbb{N}$. Then, by condition (a), we get

$$
\begin{aligned}
& \lim _{k \rightarrow \infty} \gamma\left(1-\gamma\|A\|^{2}\right)\left\|A x_{m_{k}}-B y_{m_{k}}\right\|^{2} \\
= & \lim _{k \rightarrow \infty} \gamma\left(1-\gamma\|B\|^{2}\right)\left\|A x_{m_{k}+1}-B y_{m_{k}}\right\|^{2}=0 .
\end{aligned}
$$

By the same argument, as in Case 1, we arrive at

$$
\limsup _{k \rightarrow \infty}\left\langle\left(x_{m_{k}+1}, y_{m_{k}+1}\right)-(\hat{x}, \hat{y}),(u, w)-(\hat{x}, \hat{y})\right\rangle \leq 0 .
$$


It follows from (3.17) that

$$
\begin{aligned}
E_{m_{k}+1}(\hat{x}, \hat{y}) \leq & \left(1-\alpha_{m_{k}}\right) E_{m_{k}}(\hat{x}, \hat{y}) \\
& +\alpha_{m_{k}}\left[\gamma\left(1-\gamma\|A\|^{2}\right)\left\|A x_{m_{k}}-B y_{m_{k}}\right\|^{2}\right. \\
& +\gamma\left(1-\gamma\|B\|^{2}\right)\left\|A x_{m_{k}+1}-B y_{m_{k}}\right\|^{2} \\
& \left.+2\left\langle\left(x_{m_{k}+1}, y_{m_{k}+1}\right)-(\hat{x}, \hat{y}),(u, w)-(\hat{x}, \hat{y})\right\rangle\right] .
\end{aligned}
$$

Since $E_{m_{k}}(\hat{x}, \hat{y}) \leq E_{m_{k}+1}(\hat{x}, \hat{y})$, we obtain

$$
\begin{aligned}
\alpha_{m_{k}} E_{m_{k}}(\hat{x}, \hat{y}) \leq & E_{m_{k}}(\hat{x}, \hat{y})-E_{m_{k}+1}(\hat{x}, \hat{y}) \\
& +\alpha_{m_{k}}\left[\gamma\left(1-\gamma\|A\|^{2}\right)\left\|A x_{m_{k}}-B y_{m_{k}}\right\|^{2}\right. \\
& +\gamma\left(1-\gamma\|B\|^{2}\right)\left\|A x_{m_{k}+1}-B y_{m_{k}}\right\|^{2} \\
& \left.+2\left\langle\left(x_{m_{k}+1}, y_{m_{k}+1}\right)-(\hat{x}, \hat{y}),(u, w)-(\hat{x}, \hat{y})\right\rangle\right] \\
\leq & \alpha_{m_{k}}\left[\gamma\left(1-\gamma\|A\|^{2}\right)\left\|A x_{m_{k}}-B y_{m_{k}}\right\|^{2}\right. \\
& +\gamma\left(1-\gamma\|B\|^{2}\right)\left\|A x_{m_{k}+1}-B y_{m_{k}}\right\|^{2} \\
& \left.+2\left\langle\left(x_{m_{k}+1}, y_{m_{k}+1}\right)-(\hat{x}, \hat{y}),(u, w)-(\hat{x}, \hat{y})\right\rangle\right] .
\end{aligned}
$$

In particular, since $\alpha_{m_{k}}>0$, we obtain

$$
\begin{aligned}
E_{m_{k}}(\hat{x}, \hat{y}) \leq & \gamma\left(1-\gamma\|A\|^{2}\right)\left\|A x_{m_{k}}-B y_{m_{k}}\right\|^{2} \\
& +\gamma\left(1-\gamma\|B\|^{2}\right)\left\|A x_{m_{k}+1}-B y_{m_{k}}\right\|^{2} \\
& +2\left\langle\left(x_{m_{k}+1}, y_{m_{k}+1}\right)-(\hat{x}, \hat{y}),(u, w)-(\hat{x}, \hat{y})\right\rangle .
\end{aligned}
$$

In view of (3.26)-(3.30), we deduce that

$$
\lim _{k \rightarrow \infty} E_{m_{k}}(\hat{x}, \hat{y})=0
$$

This, together with (3.28), implies that

$$
\lim _{k \rightarrow \infty} E_{m_{k}+1}(\hat{x}, \hat{y})=0 .
$$

On the other hand, we have $E_{k}(\hat{x}, \hat{y}) \leq E_{m_{k}+1}(\hat{x}, \hat{y})$ for all $k \in \mathbb{N}$ which implies that $E_{k}(\hat{x}, \hat{y}) \rightarrow 0$ as $k \rightarrow \infty$ and hence $\left(x_{m_{k}}, y_{m_{k}}\right) \rightarrow(\hat{x}, \hat{y})$ as $k \rightarrow \infty$. Thus, we have $\left(x_{n}, y_{n}\right) \rightarrow(\hat{x}, \hat{y})$ as $n \rightarrow \infty$. This completes the proof.

Theorem 3.2. Let $H_{1}, H_{2}$ and $H_{3}$ be real Hilbert spaces, $C \subset H_{1}, Q \subset H_{2}$ be two nonempty, closed and convex sets. Let $A: H_{1} \rightarrow H_{3}, B: H_{2} \rightarrow H_{3}$ be two bounded linear operators. Assume that $\zeta \in(0,1)$ and $\gamma \in\left(0, \min \left\{\frac{1}{\|A\|^{2}}, \frac{1}{\|B\|^{2}}\right\}\right)$. 
Let $\left\{S_{j}\right\}_{j \in \mathbb{N}}$ be an infinite family of quasi-nonexpansive mappings from $H_{1}$ into itself and $\left\{T_{j}\right\}_{j \in \mathbb{N}}$ be an infinite family of quasi-nonexpansive mappings from $\mathrm{H}_{2}$ into itself such that $\cap_{j=1}^{\infty} F\left(S_{j}\right)=C$ and $\cap_{j=1}^{\infty} F\left(T_{j}\right)=Q$. Assume that for each $j \in \mathbb{N}$, $S_{j}-I$ and $T_{j}-I$ are demiclosed at zero. Let $(w, z) \in H_{1} \times H_{2}$ be fixed. Let $\left\{\alpha_{n}\right\}_{n \in \mathbb{N}},\left\{\beta_{n, j}\right\}_{n \in \mathbb{N}, j \in \mathbb{N} \cup\{0\}}$ and $\left\{\delta_{n, j}\right\}_{n \in \mathbb{N}, j \in \mathbb{N} \cup\{0\}}$ be sequences in $[0,1]$ satisfying the following control conditions:

(a) $\lim _{n \rightarrow \infty} \alpha_{n}=0$;

(b) $\sum_{n=1}^{\infty} \alpha_{n}=\infty$;

(c) $\beta_{n, 0}+\sum_{j=1}^{\infty} \beta_{n, j}=1, \forall n \in \mathbb{N}$;

(d) $\liminf _{n \rightarrow \infty} \beta_{n, 0} \beta_{n, j}>0, \forall j \in \mathbb{N}$;

(e) $\delta_{n, 0}+\sum_{j=1}^{\infty} \delta_{n, j}=1, \forall n \in \mathbb{N}$, and $\liminf _{n \rightarrow \infty} \delta_{n, 0} \delta_{n, j}>0, \forall j \in \mathbb{N}$.

Let $\left\{x_{n}\right\}_{n \in \mathbb{N}}$ be a sequence generated by

$$
\left\{\begin{array}{l}
x_{0} \in H_{1}, y_{0} \in H_{2} \quad \text { chosen arbitrarily, } \\
u_{n}=\beta_{n, 0} x_{n}+\sum_{j=1}^{\infty} \beta_{n, j} S_{j} x_{n} \\
v_{n}=x_{n}-\gamma A^{*}\left(A x_{n}-B y_{n}\right) \\
w_{n}=\zeta u_{n}+(1-\zeta) v_{n} \\
s_{n}=\delta_{n, 0} y_{n}+\sum_{j=1}^{\infty} \delta_{n, j} T_{j} y_{n} \\
t_{n}=y_{n}+\gamma B^{*}\left(A x_{n+1}-B y_{n}\right) \\
z_{n}=\zeta s_{n}+(1-\zeta) t_{n} \\
x_{n+1}=\alpha_{n} w+\left(1-\alpha_{n}\right) w_{n} \\
y_{n+1}=\alpha_{n} z+\left(1-\alpha_{n}\right) z_{n}
\end{array}\right.
$$

If $\Omega:=\{(x, y) \in C \times Q: A x=B y\} \neq \varnothing$, then the sequence $\left\{\left(x_{n}, y_{n}\right)\right\}_{n \in \mathbb{N}}$ defined in (3.31) converges strongly to $P_{\Omega}(w, z)$, where $P_{\Omega}$ is the metric projection from $H_{1} \times H_{2}$ onto $\Omega$.

Proof. We divide the proof into several steps. It is clear that $\Omega$ is a closed and convex subset of $C \times Q$. Set

$$
(\hat{x}, \hat{y})=P_{\Omega}(w, z) .
$$

Step 1. We prove that the sequences $\left\{x_{n}\right\}_{n \in \mathbb{N}},\left\{u_{n}\right\}_{n \in \mathbb{N}},\left\{v_{n}\right\}_{n \in \mathbb{N}},\left\{w_{n}\right\}_{n \in \mathbb{N}}$, $\left\{y_{n}\right\}_{n \in \mathbb{N}},\left\{s_{n}\right\}_{n \in \mathbb{N}},\left\{t_{n}\right\}_{n \in \mathbb{N}},\left\{z_{n}\right\}_{n \in \mathbb{N}},\left\{A x_{n}\right\}_{n \in \mathbb{N}}$ and $\left\{B y_{n}\right\}_{n \in \mathbb{N}}$ are bounded. We first show that $\left\{x_{n}\right\}_{n \in \mathbb{N}}$ is bounded. Let $\left(x^{*}, y^{*}\right) \in \Omega$ be arbitrarily chosen. Then $x^{*} \in C, y^{*} \in Q$ and $A x^{*}=B y^{*}$. In view of Lemma 2.6 there exists a continuous, strictly increasing and convex function $h_{1}:[0, \infty) \rightarrow[0, \infty)$ such that 


$$
\begin{aligned}
& \left\|u_{n}-x^{*}\right\|^{2} \\
= & \left\|\beta_{n, 0} x_{n}+\sum_{j=1}^{\infty} \beta_{n, j} S_{j} x_{n}-x^{*}\right\|^{2} \\
\leq & \beta_{n, 0}\left\|x_{n}-x^{*}\right\|^{2}+\sum_{j=1}^{\infty} \beta_{n, j}\left\|S_{j} x_{n}-x^{*}\right\|^{2}-\beta_{n, 0} \beta_{n, j} h_{1}\left(\left\|x_{n}-S_{j} x_{n}\right\|\right) \\
\leq & \beta_{n, 0}\left\|x_{n}-x^{*}\right\|^{2}+\sum_{j=1}^{\infty} \beta_{n, j}\left\|x_{n}-x^{*}\right\|^{2}-\beta_{n, 0} \beta_{n, j} h_{1}\left(\left\|x_{n}-S_{j} x_{n}\right\|\right) \\
= & \left\|x_{n}-x^{*}\right\|^{2}-\beta_{n, 0} \beta_{n, j} h_{1}\left(\left\|x_{n}-S_{j} x_{n}\right\|\right), \quad \forall j \in \mathbb{N} .
\end{aligned}
$$

In view of Lemma 2.2(i) and (3.31), we have

$$
\begin{aligned}
& \left\|v_{n}-x^{*}\right\|^{2} \\
= & \left\|x_{n}-\gamma A^{*}\left(A x_{n}-B y_{n}\right)-x^{*}\right\|^{2} \\
= & \left\|x_{n}-x^{*}\right\|^{2}+\gamma^{2}\left\|A^{*}\left(A x_{n}-B y_{n}\right)\right\|^{2}-2 \gamma\left\langle A^{*}\left(A x_{n}-B y_{n}\right), x_{n}-x^{*}\right\rangle \\
\leq & \left\|x_{n}-x^{*}\right\|^{2}+\gamma^{2}\|A\|^{2}\left\|A x_{n}-B y_{n}\right\|^{2}-2 \gamma\left\langle A x_{n}-B y_{n}, A x_{n}-A x^{*}\right\rangle .
\end{aligned}
$$

Let $\vartheta_{n}:=2 \gamma\left\langle A x_{n}-B y_{n}, A x_{n}-A x^{*}\right\rangle$. Then we obtain

$$
\vartheta_{n}=2 \gamma\left(\left\|A x_{n}-B y_{n}\right\|^{2}+\left\langle A x_{n}-B y_{n}, B y_{n}-A x^{*}\right\rangle\right) .
$$

This implies that

$$
\begin{aligned}
& \left\|v_{n}-x^{*}\right\|^{2} \\
\leq & \left\|x_{n}-x^{*}\right\|^{2}-2 \gamma\left\langle A x_{n}-B y_{n}, B y_{n}-A x^{*}\right\rangle-\gamma\left(2-\gamma\|A\|^{2}\right)\left\|A x_{n}-B y_{n}\right\|^{2} .
\end{aligned}
$$

Similarly, in view of Lemma 2.6 there exists a continuous, strictly increasing and convex function $h_{2}:[0, \infty) \rightarrow[0, \infty)$ such that

$$
\left\|s_{n}-y^{*}\right\|^{2} \leq\left\|y_{n}-y^{*}\right\|^{2}-\delta_{n, 0} \delta_{n, j} h_{2}\left(\left\|y_{n}-T_{j} y_{n}\right\|\right), \quad \forall j \in \mathbb{N}
$$

and

$$
\begin{aligned}
\left\|t_{n}-y^{*}\right\|^{2} \leq & \left\|y_{n}-y^{*}\right\|^{2}+2 \gamma\left\langle A x_{n+1}-B y_{n}, A x_{n+1}-B y^{*}\right\rangle \\
& -\gamma\left(2-\gamma\|B\|^{2}\right)\left\|A x_{n+1}-B y_{n}\right\|^{2} .
\end{aligned}
$$

On the other hand, since $A x^{*}=B y^{*}$, we have

$$
\begin{aligned}
& 2\left\langle A x_{n}-B y_{n}, B y_{n}-A x^{*}\right\rangle \\
= & -\left\|A x_{n}-B y_{n}\right\|^{2}-\left\|B y_{n}-A x^{*}\right\|^{2}+\left\|A x_{n}-B y^{*}\right\|^{2} \\
= & -\left\|A x_{n}-B y_{n}\right\|^{2}-\left\|B y_{n}-B y^{*}\right\|^{2}+\left\|A x_{n}-A x^{*}\right\|^{2}
\end{aligned}
$$


and

$$
\begin{aligned}
& 2\left\langle B y_{n}-A x_{n+1}, A x_{n+1}-B y^{*}\right\rangle \\
= & -\left\|B y_{n}-A x_{n+1}\right\|^{2}-\left\|A x_{n+1}-B y^{*}\right\|^{2}+\left\|B y_{n}-A x^{*}\right\|^{2} \\
= & -\left\|B y_{n}-A x_{n+1}\right\|^{2}-\left\|A x_{n+1}-A x^{*}\right\|^{2}+\left\|B y_{n}-B y^{*}\right\|^{2}
\end{aligned}
$$

It follows from (3.32)-(3.39) that

$$
\begin{aligned}
\left\|v_{n}-x^{*}\right\|^{2} \leq & \left\|x_{n}-x^{*}\right\|^{2}+\gamma\left\|A x_{n}-B y_{n}\right\|^{2}+\gamma\left\|B y_{n}-B y^{*}\right\|^{2} \\
& -\gamma\left\|A x_{n}-A x^{*}\right\|^{2}-\gamma\left(2-\gamma\|A\|^{2}\right)\left\|A x_{n}-B y_{n}\right\|^{2}
\end{aligned}
$$

and

$$
\begin{aligned}
\left\|t_{n}-y^{*}\right\|^{2} \leq & \left\|y_{n}-y^{*}\right\|^{2}+\gamma\left\|A x_{n+1}-B y_{n}\right\|^{2}+\gamma\left\|A x_{n+1}-A x^{*}\right\|^{2} \\
& -\gamma\left\|B y_{n}-B y^{*}\right\|^{2}-\gamma\left(2-\gamma\|B\|^{2}\right)\left\|A x_{n+1}-B y_{n}\right\|^{2}
\end{aligned}
$$

This, together with (3.31), implies that

$$
\begin{aligned}
& \left\|w_{n}-x^{*}\right\|^{2} \\
= & \left\|\zeta u_{n}+(1-\zeta) v_{n}-x^{*}\right\|^{2} \\
\leq & \zeta\left\|u_{n}-x^{*}\right\|^{2}+(1-\zeta)\left\|v_{n}-x^{*}\right\|^{2}-\zeta(1-\zeta)\left\|u_{n}-v_{n}\right\|^{2} \\
\leq & \zeta\left[\left\|x_{n}-x^{*}\right\|^{2}-\beta_{n, 0} \beta_{n, j} h_{1}\left(\left\|x_{n}-S_{j} x_{n}\right\|\right)\right] \\
& +(1-\zeta)\left[\left\|x_{n}-x^{*}\right\|^{2}+\gamma\left\|A x_{n}-B y_{n}\right\|^{2}\right. \\
& +\gamma\left\|B y_{n}-B y^{*}\right\|^{2}-\gamma\left\|A x_{n}-A x^{*}\right\|^{2} \\
& \left.-\gamma\left(2-\gamma\|A\|^{2}\right)\left\|A x_{n}-B y_{n}\right\|^{2}\right]-\zeta(1-\zeta)\left\|u_{n}-v_{n}\right\|^{2} \\
= & \left\|x_{n}-x^{*}\right\|^{2}+(1-\zeta)\left[\gamma\left\|A x_{n}-B y_{n}\right\|^{2}+\gamma\left\|B y_{n}-B y^{*}\right\|^{2}\right] \\
& -(1-\zeta)\left[\gamma\left\|A x_{n}-A x^{*}\right\|^{2}+\gamma\left(2-\gamma\|A\|^{2}\right)\left\|A x_{n}-B y_{n}\right\|^{2}\right] \\
& -\zeta \beta_{n, 0} \beta_{n, j} h_{1}\left(\left\|x_{n}-S_{j} x_{n}\right\|\right)-\zeta(1-\zeta)\left\|u_{n}-v_{n}\right\|^{2}, \quad \forall j \in \mathbb{N} .
\end{aligned}
$$

It follows from (3.31), (3.36), (3.41) and (3.37) that

$$
\begin{aligned}
& \left\|z_{n}-y^{*}\right\|^{2} \\
= & \left\|\zeta s_{n}+(1-\zeta) t_{n}-y^{*}\right\|^{2} \\
\leq & \zeta\left\|s_{n}-y^{*}\right\|^{2}+(1-\zeta)\left\|t_{n}-y^{*}\right\|^{2}-\zeta(1-\zeta)\left\|s_{n}-t_{n}\right\|^{2} \\
\leq & \zeta\left[\left\|y_{n}-y^{*}\right\|^{2}-\delta_{n, 0} \delta_{n, j} h_{2}\left(\left\|y_{n}-T_{j} y_{n}\right\|\right)\right] \\
& +(1-\zeta)\left[\left\|y_{n}-y^{*}\right\|^{2}+\gamma\left\|B y_{n}-A x_{n+1}\right\|^{2}+\gamma\left\|A x_{n+1}-A x^{*}\right\|^{2}\right. \\
& \left.-\gamma\left\|B y_{n}-B y^{*}\right\|^{2}-\gamma\left(2-\gamma\|B\|^{2}\right)\left\|A x_{n+1}-B y_{n}\right\|^{2}\right] \\
& -\zeta(1-\zeta)\left\|s_{n}-t_{n}\right\|^{2} \\
= & \left\|y_{n}-y^{*}\right\|^{2}+(1-\zeta)\left[\gamma\left\|B y_{n}-A x_{n+1}\right\|^{2}+\gamma\left\|A x_{n+1}-A x^{*}\right\|^{2}\right] \\
& -(1-\zeta)\left[\gamma\left\|B y_{n}-B y^{*}\right\|^{2}+\gamma\left(2-\gamma\|B\|^{2}\right)\left\|A x_{n+1}-B y_{n}\right\|^{2}\right] \\
& -\zeta \delta_{n, 0} \delta_{n, j} h_{2}\left(\left\|y_{n}-T_{j} y_{n}\right\|\right)-\zeta(1-\zeta)\left\|s_{n}-t_{n}\right\|^{2}, \quad \forall j \in \mathbb{N} .
\end{aligned}
$$


Adding the two inequalities (3.42) and (3.43), we conclude that

$$
\begin{aligned}
& \left\|w_{n}-x^{*}\right\|^{2}+\left\|z_{n}-y^{*}\right\|^{2} \\
\leq & \left\|x_{n}-x^{*}\right\|^{2}+\left\|y_{n}-y^{*}\right\|^{2} \\
& -\gamma(1-\zeta)\left\|A x_{n}-A x^{*}\right\|^{2}+\gamma(1-\zeta)\left\|A x_{n+1}-A x^{*}\right\|^{2} \\
& -\gamma(1-\zeta)\left(1-\gamma\|A\|^{2}\right)\left\|A x_{n}-B y_{n}\right\|^{2} \\
& -\gamma(1-\zeta)\left(1-\gamma\|B\|^{2}\right)\left\|A x_{n+1}-B y_{n}\right\|^{2} \\
& -\zeta\left[\beta_{n, 0} \beta_{n, j} h_{1}\left(\left\|x_{n}-S_{j} x_{n}\right\|\right)+\delta_{n, 0} \delta_{n, j} h_{2}\left(\left\|y_{n}-T_{j} y_{n}\right\|\right)\right] \\
& -\zeta(1-\zeta)\left[\left\|u_{n}-v_{n}\right\|^{2}+\left\|s_{n}-t_{n}\right\|^{2}\right], \quad \forall j \in \mathbb{N}
\end{aligned}
$$

In view of (3.31) and (3.44), we obtain

$$
\begin{aligned}
& \left\|x_{n+1}-x^{*}\right\|^{2}+\left\|y_{n+1}-y^{*}\right\|^{2} \\
= & \left\|\alpha_{n} w+\left(1-\alpha_{n}\right) w_{n}-x^{*}\right\|^{2} \\
& +\left\|\alpha_{n} z+\left(1-\alpha_{n}\right) z_{n}-y^{*}\right\|^{2} \\
\leq & \alpha_{n}\left\|w-x^{*}\right\|^{2}+\left(1-\alpha_{n}\right)\left\|w_{n}-x^{*}\right\|^{2} \\
& +\alpha_{n}\left\|z-y^{*}\right\|^{2}+\left(1-\alpha_{n}\right)\left\|z_{n}-y^{*}\right\|^{2} \\
= & \alpha_{n}\left[\left\|w-x^{*}\right\|^{2}+\left\|z-y^{*}\right\|^{2}\right]+\left(1-\alpha_{n}\right)\left[\left\|w_{n}-x^{*}\right\|^{2}+\left\|z_{n}-y^{*}\right\|^{2}\right] \\
\leq & \alpha_{n}\left[\left\|w-x^{*}\right\|^{2}+\left\|z-y^{*}\right\|^{2}\right]+\left(1-\alpha_{n}\right)\left[\left\|x_{n}-x^{*}\right\|^{2}+\left\|y_{n}-y^{*}\right\|^{2}\right. \\
& -\gamma(1-\zeta)\left\|A x_{n}-A x^{*}\right\|^{2}+\gamma(1-\zeta)\left\|A x_{n+1}-A x^{*}\right\|^{2} \\
& -\gamma(1-\zeta)\left(1-\gamma\|A\|^{2}\right)\left\|A x_{n}-B y_{n}\right\|^{2} \\
& -\gamma(1-\zeta)\left(1-\gamma\|B\|^{2}\right)\left\|A x_{n+1}-B y_{n}\right\|^{2} \\
& -\zeta\left[\beta_{n, 0} \beta_{n, j} h_{1}\left(\left\|x_{n}-S_{j} x_{n}\right\|\right)+\delta_{n, 0} \delta_{n, j} h_{2}\left(\left\|y_{n}-T_{j} y_{n}\right\|\right)\right] \\
& \left.-\zeta(1-\zeta)\left[\left\|u_{n}-v_{n}\right\|^{2}+\left\|s_{n}-t_{n}\right\|^{2}\right]\right] \\
\leq & \alpha_{n}\left[\left\|w-x^{*}\right\|^{2}+\left\|z-y^{*}\right\|^{2}\right]+\gamma(1-\zeta)\left\|A x_{n+1}-A x^{*}\right\|^{2} \\
& +\left(1-\alpha_{n}\right)\left[\left\|x_{n}-x^{*}\right\|^{2}+\left\|y_{n}-y^{*}\right\|^{2}\right. \\
& \left.-\gamma(1-\zeta)\left\|A x_{n}-A x^{*}\right\|^{2}\right] \\
& -\left(1-\alpha_{n}\right)\left[\gamma(1-\zeta)\left(1-\gamma\|A\|^{2}\right)\left\|A x_{n}-B y_{n}\right\|^{2}\right. \\
& +\gamma(1-\zeta)\left(1-\gamma\|B\|^{2}\right)\left\|A x_{n+1}-B y_{n}\right\|^{2} \\
& +\zeta\left[\beta_{n, 0} \beta_{n, j} h_{1}\left(\left\|x_{n}-S_{j} x_{n}\right\|\right)+\delta_{n, 0} \delta_{n, j} h_{2}\left(\left\|y_{n}-T_{j} y_{n}\right\|\right)\right] \\
& \left.+\zeta(1-\zeta)\left[\left\|u_{n}-v_{n}\right\|^{2}+\left\|s_{n}-t_{n}\right\|^{2}\right]\right], \quad \forall j \in \mathbb{N}
\end{aligned}
$$

Put

$$
G_{n}\left(x^{*}, y^{*}\right):=\left\|x_{n}-x^{*}\right\|^{2}+\left\|y_{n}-y^{*}\right\|^{2}-\gamma(1-\zeta)\left\|A x_{n}-A x^{*}\right\|^{2} .
$$

We note that

$$
\gamma(1-\zeta)\left\|A x_{n}-A x^{*}\right\|^{2} \leq \gamma(1-\zeta)\|A\|^{2}\left\|x_{n}-x^{*}\right\|^{2} .
$$


Therefore

$$
G_{n}\left(x^{*}, y^{*}\right) \geq\left(1-\gamma(1-\zeta)\|A\|^{2}\right)\left\|x_{n}-x^{*}\right\|^{2}+\left\|y_{n}-y^{*}\right\|^{2} \geq 0 .
$$

In view of (3.45)-(3.46), we conclude that

$$
\begin{aligned}
& G_{n+1}\left(x^{*}, y^{*}\right) \\
\leq & \left(1-\alpha_{n}\right) G_{n}\left(x^{*}, y^{*}\right)+\alpha_{n}\left[\left\|w-x^{*}\right\|^{2}+\left\|z-y^{*}\right\|^{2}\right] \\
& -\left(1-\alpha_{n}\right)\left[\gamma(1-\zeta)\left(1-\gamma\|A\|^{2}\right)\left\|A x_{n}-B y_{n}\right\|^{2}\right. \\
& +\gamma(1-\zeta)\left(1-\gamma\|B\|^{2}\right)\left\|A x_{n+1}-B y_{n}\right\|^{2} \\
& +\zeta\left[\beta_{n, 0} \beta_{n, j} h_{1}\left(\left\|x_{n}-S_{j} x_{n}\right\|\right)+\delta_{n, 0} \delta_{n, j} h_{2}\left(\left\|y_{n}-T_{j} y_{n}\right\|\right)\right] \\
& \left.+\zeta(1-\zeta)\left[\left\|u_{n}-v_{n}\right\|^{2}+\left\|s_{n}-t_{n}\right\|^{2}\right]\right], \quad \forall j \in \mathbb{N} .
\end{aligned}
$$

This implies that

$$
\begin{aligned}
G_{n+1}\left(x^{*}, y^{*}\right) & \leq\left(1-\alpha_{n}\right) G_{n}\left(x^{*}, y^{*}\right)+\alpha_{n}\left[\left\|u-x^{*}\right\|^{2}+\left\|w-y^{*}\right\|^{2}\right] \\
& \leq \max \left\{G_{n}\left(x^{*}, y^{*}\right),\left\|u-x^{*}\right\|^{2}+\left\|w-y^{*}\right\|^{2}\right\}
\end{aligned}
$$

By induction, we obtain

$$
G_{n+1}\left(x^{*}, y^{*}\right) \leq \max \left\{G_{1}\left(x^{*}, y^{*}\right),\left\|u-x^{*}\right\|^{2}+\left\|w-y^{*}\right\|^{2}\right\}
$$

for all $n \in \mathbb{N}$. This implies that the sequence $\left\{G_{n}\left(x^{*}, y^{*}\right)\right\}_{n \in \mathbb{N}}$ is bounded and hence, in view of (3.47), the sequences $\left\{x_{n}\right\}_{n \in \mathbb{N}}$ and $\left\{y_{n}\right\}_{n \in \mathbb{N}}$ are bounded. This, together with (3.31), implies that the sequences $\left\{v_{n}\right\}_{n \in \mathbb{N}},\left\{w_{n}\right\}_{n \in \mathbb{N}},\left\{s_{n}\right\}_{n \in \mathbb{N}},\left\{t_{n}\right\}_{n \in \mathbb{N}},\left\{z_{n}\right\}_{n \in \mathbb{N}}$, $\left\{A x_{n}\right\}_{n \in \mathbb{N}}$ and $\left\{B y_{n}\right\}_{n \in \mathbb{N}}$ are bounded too.

In view of (3.48), we conclude that

$$
\begin{aligned}
& G_{n+1}(\hat{x}, \hat{y}) \\
\leq & \left(1-\alpha_{n}\right) G_{n}(\hat{x}, \hat{y})+\alpha_{n}\left[\|w-\hat{x}\|^{2}+\|z-\hat{y}\|^{2}\right. \\
& +\gamma\left(1-\gamma\|A\|^{2}\right)\left\|A x_{n}-B y_{n}\right\|^{2} \\
& +\gamma\left(1-\gamma\|B\|^{2}\right)\left\|A x_{n+1}-B y_{n}\right\|^{2} \\
& +\gamma\left(1-\gamma\|A\|^{2}\right)\left\|A x_{n}-B y_{n}\right\|^{2}+\gamma\left(1-\gamma\|B\|^{2}\right)\left\|A x_{n+1}-B y_{n}\right\|^{2} \\
& +\zeta\left[\beta_{n, 0} \beta_{n, j} h_{1}\left(\left\|x_{n}-S_{j} x_{n}\right\|\right)+\delta_{n, 0} \delta_{n, j} h_{2}\left(\left\|y_{n}-T_{j} y_{n}\right\|\right)\right] \\
& \left.+\zeta(1-\zeta)\left[\left\|u_{n}-v_{n}\right\|^{2}+\left\|s_{n}-t_{n}\right\|^{2}\right]\right], \quad \forall j \in \mathbb{N} .
\end{aligned}
$$

Let

$$
\begin{aligned}
M_{4}:= & \sup \left\{\|w-\hat{x}\|^{2}+\|z-\hat{y}\|^{2}+\gamma(1-\zeta)\left(1-\gamma\|A\|^{2}\right)\left\|A x_{n}-B y_{n}\right\|^{2}\right. \\
& +\gamma(1-\zeta)\left(1-\gamma\|B\|^{2}\right)\left\|A x_{n+1}-B y_{n}\right\|^{2} \\
& +\zeta\left[\beta_{n, 0} \beta_{n, j} h_{1}\left(\left\|x_{n}-S_{j} x_{n}\right\|\right)+\delta_{n, 0} \delta_{n, j} h_{2}\left(\left\|y_{n}-T_{j} y_{n}\right\|\right)\right] \\
& \left.+\zeta(1-\zeta)\left[\left\|u_{n}-v_{n}\right\|^{2}+\left\|s_{n}-t_{n}\right\|^{2}\right]: n \in \mathbb{N} \cup\{0\}, j \in \mathbb{N}\right\} .
\end{aligned}
$$


It follows from (3.48) and (3.49) that

$$
\begin{aligned}
& \zeta(1-\zeta)\left[\left\|u_{n}-v_{n}\right\|^{2}+\left\|s_{n}-t_{n}\right\|^{2}\right] \\
& +\zeta\left[\beta_{n, 0} \beta_{n, j} h_{1}\left(\left\|x_{n}-S_{j} x_{n}\right\|\right)+\delta_{n, 0} \delta_{n, j} h_{2}\left(\left\|y_{n}-T_{j} y_{n}\right\|\right)\right] \\
& +\gamma(1-\zeta)\left(1-\gamma\|A\|^{2}\right)\left\|A x_{n}-B y_{n}\right\|^{2} \\
& +\gamma(1-\zeta)\left(1-\gamma\|B\|^{2}\right)\left\|A x_{n+1}-B y_{n}\right\|^{2} \\
\leq & G_{n}(\hat{x}, \hat{y})-G_{n+1}(\hat{x}, \hat{y})+\alpha_{n} M_{4}, \quad \forall j \in \mathbb{N} .
\end{aligned}
$$

Step 2. We prove that for any $n \in \mathbb{N}$

$$
\begin{aligned}
G_{n+1}(\hat{x}, \hat{y}) \leq & \left(1-\alpha_{n}\right) G_{n}(\hat{x}, \hat{y}) \\
& +\alpha_{n}\left[\gamma(1-\zeta)\left(1-\gamma\|A\|^{2}\right)\left\|A x_{n}-B y_{n}\right\|^{2}\right. \\
& +\gamma(1-\zeta)\left(1-\gamma\|B\|^{2}\right)\left\|A x_{n+1}-B y_{n}\right\|^{2} \\
& +\zeta\left[\beta_{n, 0} \beta_{n, j} h_{1}\left(\left\|x_{n}-S_{j} x_{n}\right\|\right)+\delta_{n, 0} \delta_{n, j} h_{2}\left(\left\|y_{n}-T_{j} y_{n}\right\|\right)\right] \\
& +\zeta(1-\zeta)\left[\left\|x_{n}-u_{n}\right\|^{2}+\left\|s_{n}-w_{n}\right\|^{2}\right] \\
& \left.+2\left\langle\left(x_{n+1}, y_{n+1}\right)-(\hat{x}, \hat{y}),(w, z)-(\hat{x}, \hat{y})\right\rangle\right], \quad \forall j \in \mathbb{N} .
\end{aligned}
$$

Let us show (3.51). In view of Lemma 2.2(iii), we obtain

$$
\begin{aligned}
& G_{n+1}(\hat{x}, \hat{y}) \\
= & \left\|x_{n+1}-\hat{x}\right\|^{2}+\left\|y_{n+1}-\hat{y}\right\|^{2}-\gamma(1-\zeta)\left\|A x_{n+1}-A \hat{x}\right\|^{2} \\
= & \left\|\left(x_{n+1}, y_{n+1}\right)-(\hat{x}, \hat{y})\right\|^{2}-\gamma(1-\zeta)\left\|A x_{n+1}-A \hat{x}\right\|^{2} \\
= & \left\|\alpha_{n}(w, z)+\left(1-\alpha_{n}\right)\left(w_{n}, z_{n}\right)-(\hat{x}, \hat{y})\right\|^{2}-\gamma(1-\zeta)\left\|A x_{n+1}-A \hat{x}\right\|^{2} \\
\leq & \left(1-\alpha_{n}\right)\left\|\left(w_{n}, z_{n}\right)-(\hat{x}, \hat{y})\right\|^{2} \\
& +2 \alpha_{n}\left\langle\left(x_{n+1}, y_{n+1}\right)-(\hat{x}, \hat{y}),(w, z)-(\hat{x}, \hat{y})\right\rangle-\gamma(1-\zeta)\left\|A x_{n+1}-A \hat{x}\right\|^{2} \\
= & \left(1-\alpha_{n}\right)\left[\|\left(w_{n}-\hat{x}\left\|^{2}+\right\| z_{n}-\hat{y} \|^{2}\right]\right. \\
& +2 \alpha_{n}\left\langle\left(x_{n+1}, y_{n+1}\right)-(\hat{x}, \hat{y}),(w, z)-(\hat{x}, \hat{y})\right\rangle-\gamma(1-\zeta)\left\|A x_{n+1}-A \hat{x}\right\|^{2} \\
\leq & \left(1-\alpha_{n}\right)\left[\left\|x_{n}-x^{*}\right\|^{2}+\left\|y_{n}-y^{*}\right\|^{2}\right. \\
& -\gamma(1-\zeta)\left\|A x_{n}-A x^{*}\right\|^{2}+\gamma(1-\zeta)\left\|A x_{n+1}-A x^{*}\right\|^{2} \\
& -\gamma(1-\zeta)\left(1-\gamma\|A\|^{2}\right)\left\|A x_{n}-B y_{n}\right\|^{2} \\
& -\gamma(1-\zeta)\left(1-\gamma\|B\|^{2}\right)\left\|A x_{n+1}-B y_{n}\right\|^{2} \\
& -\zeta\left[\beta_{n, 0} \beta_{n, j} h_{1}\left(\left\|x_{n}-S_{j} x_{n}\right\|\right)+\delta_{n, 0} \delta_{n, j} h_{2}\left(\left\|y_{n}-T_{j} y_{n}\right\|\right)\right] \\
& \left.-\zeta(1-\zeta)\left[\left\|x_{n}-u_{n}\right\|^{2}+\left\|s_{n}-w_{n}\right\|^{2}\right]\right] \\
& +2 \alpha_{n}\left\langle\left(x_{n+1}, y_{n+1}\right)-(\hat{x}, \hat{y}),(w, z)-(\hat{x}, \hat{y})\right\rangle-\gamma(1-\zeta)\left\|A x_{n+1}-A \hat{x}\right\|^{2} \\
\leq & \left(1-\alpha_{n}\right)\left[\left\|x_{n}-x^{*}\right\|^{2}+\left\|y_{n}-y^{*}\right\|^{2}-\gamma(1-\zeta)\left\|A x_{n}-A x^{*}\right\|^{2}\right] \\
& +\gamma(1-\zeta)\left\|A x_{n+1}-A x^{*}\right\|^{2} \\
& -\left(1-\alpha_{n}\right)\left[\gamma(1-\zeta)\left(1-\gamma\|A\|^{2}\right)\left\|A x_{n}-B y_{n}\right\|^{2}\right.
\end{aligned}
$$




$$
\begin{aligned}
& +\gamma(1-\zeta)\left(1-\gamma\|B\|^{2}\right)\left\|A x_{n+1}-B y_{n}\right\|^{2} \\
& +\zeta\left[\beta_{n, 0} \beta_{n, j} h_{1}\left(\left\|x_{n}-S_{j} x_{n}\right\|\right)+\delta_{n, 0} \delta_{n, j} h_{2}\left(\left\|y_{n}-T_{j} y_{n}\right\|\right)\right] \\
& \left.+\zeta(1-\zeta)\left[\left\|u_{n}-v_{n}\right\|^{2}+\left\|s_{n}-t_{n}\right\|^{2}\right]\right] \\
& +2 \alpha_{n}\left\langle\left(x_{n+1}, y_{n+1}\right)-(\hat{x}, \hat{y}),(w, z)-(\hat{x}, \hat{y})\right\rangle-\gamma(1-\zeta)\left\|A x_{n+1}-A \hat{x}\right\|^{2} \\
& =\left(1-\alpha_{n}\right) G_{n}(\hat{x}, \hat{y}) \\
& +\left(1-\alpha_{n}\right)\left[\gamma(1-\zeta)\left(1-\gamma\|A\|^{2}\right)\left\|A x_{n}-B y_{n}\right\|^{2}\right. \\
& +\gamma(1-\zeta)\left(1-\gamma\|B\|^{2}\right)\left\|A x_{n+1}-B y_{n}\right\|^{2} \\
& +\zeta\left[\beta_{n, 0} \beta_{n, j} h_{1}\left(\left\|x_{n}-S_{j} x_{n}\right\|\right)+\delta_{n, 0} \delta_{n, j} h_{2}\left(\left\|y_{n}-T_{j} y_{n}\right\|\right)\right] \\
& \left.+\zeta(1-\zeta)\left[\left\|u_{n}-v_{n}\right\|^{2}+\left\|s_{n}-t_{n}\right\|^{2}\right]\right] \\
& +2 \alpha_{n}\left\langle\left(x_{n+1}, y_{n+1}\right)-(\hat{x}, \hat{y}),(w, z)-(\hat{x}, \hat{y})\right\rangle, \quad \forall j \in \mathbb{N} .
\end{aligned}
$$

This implies that

$$
\begin{aligned}
G_{n+1}(\hat{x}, \hat{y}) \leq & \left(1-\alpha_{n}\right) G_{n}(\hat{x}, \hat{y}) \\
& +\alpha_{n}\left[\gamma(1-\zeta)\left(1-\gamma\|A\|^{2}\right)\left\|A x_{n}-B y_{n}\right\|^{2}\right. \\
& +\gamma(1-\zeta)\left(1-\gamma\|B\|^{2}\right)\left\|A x_{n+1}-B y_{n}\right\|^{2} \\
& +\zeta\left[\beta_{n, 0} \beta_{n, j} h_{1}\left(\left\|x_{n}-S_{j} x_{n}\right\|\right)+\delta_{n, 0} \delta_{n, j} h_{2}\left(\left\|y_{n}-T_{j} y_{n}\right\|\right)\right] \\
& +\zeta(1-\zeta)\left[\left\|u_{n}-v_{n}\right\|^{2}+\left\|s_{n}-t_{n}\right\|^{2}\right] \\
& \left.+2\left\langle\left(x_{n+1}, y_{n+1}\right)-(\hat{x}, \hat{y}),(w, z)-(\hat{x}, \hat{y})\right\rangle\right], \quad \forall j \in \mathbb{N} .
\end{aligned}
$$

Thus we have (3.51).

Step 3. We prove that $\left(x_{n}, y_{n}\right) \rightarrow(\hat{x}, \hat{y})$ as $n \rightarrow \infty$. We discuss the following two possible cases:

Case 1. Suppose that there exists $n_{0} \in \mathbb{N}$ such that $\left\{G_{n}(\hat{x}, \hat{y})\right\}_{n=n_{0}}^{\infty}$ is decreasing. Then, the sequence $\left\{G_{n}(\hat{x}, \hat{y})\right\}_{n \in \mathbb{N}}$ is convergent. Thus we have $G_{n}(\hat{x}, \hat{y})-$ $G_{n+1}(\hat{x}, \hat{y}) \rightarrow 0$ as $n \rightarrow \infty$. This, together with (c), (d), (e) and (3.50), implies that

$$
\lim _{n \rightarrow \infty}\left[\gamma\left(1-\gamma\|A\|^{2}\right)\left\|A x_{n}-B y_{n}\right\|^{2}+\gamma\left(1-\gamma\|B\|^{2}\right)\left\|A x_{n+1}-B y_{n}\right\|^{2}\right]=0
$$

and for all $j \in \mathbb{N}$

$$
\begin{aligned}
& \quad \lim _{n \rightarrow \infty}\left[\zeta(1-\zeta)\left[\left\|u_{n}-v_{n}\right\|^{2}+\left\|s_{n}-t_{n}\right\|^{2}\right]\right. \\
& \left.+\zeta\left[\beta_{n, 0} \beta_{n, j} h_{1}\left(\left\|x_{n}-S_{j} x_{n}\right\|\right)+\delta_{n, 0} \delta_{n, j} h_{2}\left(\left\|y_{n}-T_{j} y_{n}\right\|\right)\right]\right]=0 .
\end{aligned}
$$

From the properties of $h_{1}$ and $h_{2}$, we conclude that

$$
\lim _{n \rightarrow \infty}\left\|x_{n}-S_{j} x_{n}\right\|=0, \quad \lim _{n \rightarrow \infty}\left\|y_{n}-T_{j} y_{n}\right\|=0, \forall j \in \mathbb{N} .
$$


On the other hand, we have

$$
\begin{aligned}
& u_{n}-x_{n}=\sum_{j=1}^{\infty} \beta_{n, j}\left(S_{j} x_{n}-x_{n}\right), x_{n}-v_{n}=\gamma A^{*}\left(A x_{n}-B y_{n}\right), \\
& w_{n}-v_{n}=\zeta\left(u_{n}-v_{n}\right), x_{n+1}-w_{n}=\alpha_{n}\left(w-w_{n}\right)
\end{aligned}
$$

and

$$
\begin{aligned}
& s_{n}-y_{n}=\sum_{j=1}^{\infty} \delta_{n, j}\left(T_{j} y_{n}-y_{n}\right), t_{n}-y_{n}=\gamma B^{*}\left(A x_{n+1}-B y_{n}\right), \\
& w_{n}-v_{n}=\zeta\left(s_{n}-t_{n}\right), y_{n+1}-z_{n}=\alpha_{n}\left(z-z_{n}\right) .
\end{aligned}
$$

Therefore

$$
\lim _{n \rightarrow \infty}\left\|x_{n+1}-x_{n}\right\|=0, \quad \lim _{n \rightarrow \infty}\left\|y_{n+1}-y_{n}\right\|=0 .
$$

Since, for any $j \in \mathbb{N}, T_{j}-I$ is demiclosed at 0 (see Definition 2.1), there exists a subsequence $\left\{x_{n_{i}}\right\}_{i \in \mathbb{N}}$ of $\left\{x_{n}\right\}_{n \in \mathbb{N}}$ converging weakly to some $u \in C$. In a similar way, we conclude that there exists a subsequence $\left\{y_{n_{i}}\right\}_{i \in \mathbb{N}}$ of $\left\{y_{n}\right\}_{n \in \mathbb{N}}$ converging weakly to some $v \in Q$. By a similar argument, as in the proof of Theorem 3.1, we can show the uniqueness of weak cluster points of the sequence $\left\{\left(x_{n}, y_{n}\right)\right\}_{n \in \mathbb{N}}$. Now, by lower semicontiuity of the squared norm and the weak convergence of $\left\{A x_{n_{i}}-B y_{n_{i}}\right\}_{i \in \mathbb{N}}$, we conclude that

$$
\|A z-B v\|^{2} \leq \liminf _{i \rightarrow \infty}\left\|A x_{n_{i}}-B y_{n_{i}}\right\|^{2}=0,
$$

which shows that $(u, v) \in \Omega$. Without loss of generality, we may assume that

$$
\begin{aligned}
& \limsup _{n \rightarrow \infty}\left\langle\left(x_{n}, y_{n}\right)-(\hat{x}, \hat{y}),(w, z)-(\hat{x}, \hat{y})\right\rangle \\
= & \lim _{i \rightarrow \infty}\left\langle\left(x_{n_{i}}, y_{n_{i}}\right)-(\hat{x}, \hat{y}),(w, z)-(\hat{x}, \hat{y})\right\rangle \\
= & \langle(u, v)-(\hat{x}, \hat{y}),(w, z)-(\hat{x}, \hat{y})\rangle .
\end{aligned}
$$

This, together with Lemma 2.1(ii), implies that

$$
\begin{aligned}
& \limsup _{n \rightarrow \infty}\left\langle\left(x_{n+1}, y_{n+1}\right)-(\hat{x}, \hat{y}),(w, z)-(\hat{x}, \hat{y})\right\rangle \\
\leq & \limsup _{n \rightarrow \infty}\left\langle\left(x_{n+1}, y_{n+1}\right)-\left(x_{n}, y_{n}\right),(w, z)-(\hat{x}, \hat{y})\right\rangle \\
& +\limsup _{n \rightarrow \infty}\left\langle\left(x_{n}, y_{n}\right)-(\hat{x}, \hat{y}),(w, z)-(\hat{x}, \hat{y})\right\rangle \\
= & \limsup _{n \rightarrow \infty}\left\langle\left(x_{n}, y_{n}\right)-(\hat{x}, \hat{y}),(w, z)-(\hat{x}, \hat{y})\right\rangle \\
= & \lim _{i \rightarrow \infty}\left\langle\left(x_{n_{i}}, y_{n_{i}}\right)-(\hat{x}, \hat{y}),(w, z)-(\hat{x}, \hat{y})\right\rangle \\
= & \langle(u, v)-(\hat{x}, \hat{y}),(w, z)-(\hat{x}, \hat{y}) \\
= & \left\langle(u, v)-P_{\Omega}(w, z),(w, z)-P_{\Omega}(w, z)\right\rangle \\
\leq & 0 .
\end{aligned}
$$


Thus we have the desired result by (3.48) and Lemma 2.4.

Case 2. Suppose that there exists a subsequence $\left\{n_{i}\right\}_{i \in \mathbb{N}}$ of $\{n\}_{n \in \mathbb{N}}$ such that

$$
G_{n_{i}}(\hat{x}, \hat{y})<G_{n_{i}+1}(\hat{x}, \hat{y})
$$

for all $i \in \mathbb{N}$. Then, by Lemma 2.5, there exists a nondecreasing sequence $\left\{m_{k}\right\}_{k \in \mathbb{N}} \subset$ $\mathbb{N}$ such that $m_{k} \rightarrow \infty$,

$$
G_{m_{k}}(\hat{x}, \hat{y}) \leq G_{m_{k}+1}(\hat{x}, \hat{y}) \quad \text { and } \quad G_{k}(\hat{x}, \hat{y}) \leq G_{m_{k}+1}(\hat{x}, \hat{y})
$$

for all $k \in \mathbb{N}$. This, together with (3.48), implies that

$$
\begin{aligned}
& \zeta(1-\zeta)\left[\left\|u_{m_{k}}-v_{m_{k}}\right\|^{2}+\left\|s_{m_{k}}-t_{m_{k}}\right\|^{2}\right] \\
& +\zeta\left[\beta_{m_{k}, 0} \beta_{m_{k}, j} h_{1}\left(\left\|x_{m_{k}}-S_{j} x_{m_{k}}\right\|\right)+\delta_{m_{k}, 0} \delta_{m_{k}, j} h_{2}\left(\left\|y_{m_{k}}-T_{j} y_{m_{k}}\right\|\right)\right] \\
& +\gamma\left(1-\gamma\|A\|^{2}\right)\left\|A x_{m_{k}}-B y_{m_{k}}\right\|^{2}+\gamma\left(1-\gamma\|B\|^{2}\right)\left\|A x_{m_{k}+1}-B y_{m_{k}}\right\|^{2} \\
& \leq G_{m_{k}}(\hat{x}, \hat{y})-G_{m_{k}+1}(\hat{x}, \hat{y})+\alpha_{m_{k}} M_{4} \\
& \leq \alpha_{m_{k}} M_{4}
\end{aligned}
$$

for all $j, k \in \mathbb{N}$. Then, by condition (i), we get

$$
\lim _{k \rightarrow \infty}\left[\gamma\left(1-\gamma\|A\|^{2}\right)\left\|A x_{m_{k}}-B y_{m_{k}}\right\|^{2}+\gamma\left(1-\gamma\|B\|^{2}\right)\left\|A x_{m_{k}+1}-B y_{m_{k}}\right\|^{2}\right]=0
$$

and

$$
\begin{aligned}
& \lim _{k \rightarrow \infty}\left[\zeta ( 1 - \zeta ) \left[\left\|u_{m_{k}}-v_{m_{k}}\right\|^{2}\right.\right. \\
& \left.+\left\|s_{m_{k}}-t_{m_{k}}\right\|^{2}\right]+\zeta\left[\beta_{m_{k}, 0} \beta_{m_{k}, j} h_{1}\left(\left\|x_{m_{k}}-S_{j} x_{m_{k}}\right\|\right)\right. \\
& \left.\left.+\delta_{m_{k}, 0} \delta_{m_{k}, j} h_{2}\left(\left\|y_{m_{k}}-T_{j} y_{m_{k}}\right\|\right)\right]\right]=0, \quad \forall j \in \mathbb{N} .
\end{aligned}
$$

By the same argument, as in Case 1, we arrive at

$$
\limsup _{k \rightarrow \infty}\left\langle\left(x_{m_{k}+1}, y_{m_{k}+1}\right)-(\hat{x}, \hat{y}),(w, z)-(\hat{x}, \hat{y})\right\rangle \leq 0 .
$$

It follows from (3.51) that

$$
\begin{aligned}
& G_{m_{k}+1}(\hat{x}, \hat{y}) \\
\leq & \left(1-\alpha_{m_{k}}\right) G_{m_{k}}(\hat{x}, \hat{y}) \\
& +\alpha_{m_{k}}\left[\zeta(1-\zeta)\left[\left\|u_{m_{k}}-v_{m_{k}}\right\|^{2}+\left\|s_{m_{k}}-t_{m_{k}}\right\|^{2}\right]\right. \\
& +\zeta\left[\beta_{m_{k}, 0} \beta_{m_{k}, j} h_{1}\left(\left\|x_{m_{k}}-S_{j} x_{m_{k}}\right\|\right)+\delta_{m_{k}, 0} \delta_{m_{k}, j} h_{2}\left(\left\|y_{m_{k}}-T_{j} y_{m_{k}}\right\|\right)\right] \\
& +\gamma\left(1-\gamma\|A\|^{2}\right)\left\|A x_{m_{k}}-B y_{m_{k}}\right\|^{2}+\gamma\left(1-\gamma\|B\|^{2}\right)\left\|A x_{m_{k}+1}-B y_{m_{k}}\right\|^{2} \\
& \left.+2\left\langle\left(x_{m_{k}+1}, y_{m_{k}+1}\right)-(\hat{x}, \hat{y}),(w, z)-(\hat{x}, \hat{y})\right\rangle\right], \quad \forall j \in \mathbb{N} .
\end{aligned}
$$


Since $G_{m_{k}}(\hat{x}, \hat{y}) \leq G_{m_{k}+1}(\hat{x}, \hat{y})$, we obtain

$$
\begin{aligned}
& \alpha_{m_{k}} G_{m_{k}}(\hat{x}, \hat{y}) \\
\leq & G_{m_{k}}(\hat{x}, \hat{y})-G_{m_{k}+1}(\hat{x}, \hat{y}) \\
& +\alpha_{m_{k}}\left[\zeta(1-\zeta)\left[\left\|u_{m_{k}}-v_{m_{k}}\right\|^{2}+\left\|s_{m_{k}}-t_{m_{k}}\right\|^{2}\right]\right. \\
& +\zeta\left[\beta_{m_{k}, 0} \beta_{m_{k}, j} h_{1}\left(\left\|x_{m_{k}}-S_{j} x_{m_{k}}\right\|\right)\right. \\
& \left.+\delta_{m_{k}, 0} \delta_{m_{k}, j} h_{2}\left(\left\|y_{m_{k}}-T_{j} y_{m_{k}}\right\|\right)\right] \\
& +\gamma\left(1-\gamma\|A\|^{2}\right)\left\|A x_{m_{k}}-B y_{m_{k}}\right\|^{2}+\gamma\left(1-\gamma\|B\|^{2}\right)\left\|A x_{m_{k}+1}-B y_{m_{k}}\right\|^{2} \\
& \left.+2\left\langle\left(x_{m_{k}+1}, y_{m_{k}+1}\right)-(\hat{x}, \hat{y}),(w, z)-(\hat{x}, \hat{y})\right\rangle\right] \\
\leq & \alpha_{m_{k}}\left[\zeta(1-\zeta)\left[\left\|u_{m_{k}}-v_{m_{k}}\right\|^{2}+\left\|s_{m_{k}}-t_{m_{k}}\right\|^{2}\right]\right. \\
& +\zeta\left[\beta_{m_{k}, 0} \beta_{m_{k}, j} h_{1}\left(\left\|x_{m_{k}}-S_{j} x_{m_{k}}\right\|\right)\right. \\
& \left.+\delta_{m_{k}, 0} \delta_{m_{k}, j} h_{2}\left(\left\|y_{m_{k}}-T_{j} y_{m_{k}}\right\|\right)\right] \\
& +\gamma\left(1-\gamma\|A\|^{2}\right)\left\|A x_{m_{k}}-B y_{m_{k}}\right\|^{2}+\gamma\left(1-\gamma\|B\|^{2}\right)\left\|A x_{m_{k}+1}-B y_{m_{k}}\right\|^{2} \\
& \left.+2\left\langle\left(x_{m_{k}+1}, y_{m_{k}+1}\right)-(\hat{x}, \hat{y}),(w, z)-(\hat{x}, \hat{y})\right\rangle\right], \quad \forall j \in \mathbb{N} .
\end{aligned}
$$

In particular, since $\alpha_{m_{k}}>0$, we obtain

$$
\begin{aligned}
& G_{m_{k}}(\hat{x}, \hat{y}) \\
\leq & \zeta(1-\zeta)\left[\left\|u_{m_{k}}-v_{m_{k}}\right\|^{2}+\left\|s_{m_{k}}-t_{m_{k}}\right\|^{2}\right] \\
& +\zeta\left[\beta_{m_{k}, 0} \beta_{m_{k}, j} h_{1}\left(\left\|x_{m_{k}}-S_{j} x_{m_{k}}\right\|\right)\right. \\
& \left.+\delta_{m_{k}, 0} \delta_{m_{k}, j} h_{2}\left(\left\|y_{m_{k}}-T_{j} y_{m_{k}}\right\|\right)\right] \\
& +\gamma\left(1-\gamma\|A\|^{2}\right)\left\|A x_{m_{k}}-B y_{m_{k}}\right\|^{2}+\gamma\left(1-\gamma\|B\|^{2}\right)\left\|A x_{m_{k}+1}-B y_{m_{k}}\right\|^{2} \\
& +2\left\langle\left(x_{m_{k}+1}, y_{m_{k}+1}\right)-(\hat{x}, \hat{y}),(w, z)-(\hat{x}, \hat{y})\right\rangle, \quad \forall j \in \mathbb{N} .
\end{aligned}
$$

In view of (3.54) and (3.60), we deduce that

$$
\lim _{k \rightarrow \infty} G_{m_{k}}(\hat{x}, \hat{y})=0
$$

This, together with (3.58), implies that

$$
\lim _{k \rightarrow \infty} G_{m_{k}+1}(\hat{x}, \hat{y})=0 .
$$

On the other hand, we have $G_{k}(\hat{x}, \hat{y}) \leq G_{m_{k}+1}(\hat{x}, \hat{y})$ for all $k \in \mathbb{N}$ which implies that $\left(x_{m_{k}}, y_{m_{k}}\right) \rightarrow(\hat{x}, \hat{y})$ as $k \rightarrow \infty$. Thus, we have $\left(x_{n}, y_{n}\right) \rightarrow(\hat{x}, \hat{y})$ as $n \rightarrow \infty$, which completes the proof. 


\section{Remark 3.1.}

(1) We provide a positive answer to open question 1.1 (Theorem 3.1) in real Hilbert spaces; furthermore, our method of proof is different from that in [32].

(2) We prove a strong convergence theorem for approximating common fixed points of a countable family of quasi-nonexpansive mappings in Hilbert spaces. Our strongly iterative sequence (3.31) is of modified Halpern-type scheme. In [32], the author studied a relaxed alternating CQ-algorithm (RACQA) with weak convergence for convex feasibility problems; they are independent of each other. Our scheme has an advantage that we do not use any projection which creates some difficulties in a practical calculation of the iterative sequence. Consequently, the above question is answered in the affirmative without using projection algorithm.

(3) Theorems 3.1 and 3.2 improve Theorem 1.1 and many results of fixed point in the literature.

(4) In most cases strong convergence is more desirable than weak convergence.

\section{Strong Convergence Theorems for Split Common Fixed-point Problems}

In the following, we first prove the following strong convergence theorem of Halpern-type for split common fixed-point problems in real Hilbert spaces.

Theorem 4.1. Let $H_{1}$ and $H_{2}$ be two real Hilbert spaces, $C \subset H_{1}, Q \subset H_{2}$ be two nonempty, closed and convex sets. Let $A: H_{1} \rightarrow H_{2}$ be a bounded linear operator, $\left\{S_{j}\right\}_{j \in \mathbb{N}}$ be an infinite family of quasi-nonexpansive mappings from $H_{1}$ into itself and $\left\{T_{j}\right\}_{j \in \mathbb{N}}$ be an infinite family of quasi-nonexpansive mappings from $\mathrm{H}_{2}$ into itself such that $\cap_{j=1}^{\infty} F\left(S_{j}\right)=C$ and $\cap_{j=1}^{\infty} F\left(T_{j}\right)=Q$. Assume that for each $j \in \mathbb{N}, S_{j}-I$ and $T_{j}-I$ are demiclosed at 0 . Let $z \in H_{1}$ be fixed. Let $\left\{\alpha_{n}\right\}_{n \in \mathbb{N}},\left\{\beta_{n, j}\right\}_{n \in \mathbb{N}, j \in \mathbb{N} \cup\{0\}}$ and $\left\{\delta_{n, j}\right\}_{n, j \in \mathbb{N}}$ be sequences in $[0,1]$ satisfying the following control conditions:

(a) $\lim _{n \rightarrow \infty} \alpha_{n}=0$;

(b) $\sum_{n=1}^{\infty} \alpha_{n}=\infty$;

(c) $\beta_{n, 0}+\sum_{j=1}^{\infty} \beta_{n, j}=1, \forall n \in \mathbb{N}$;

(d) $\liminf _{n \rightarrow \infty} \beta_{n, 0} \beta_{n, j}>0, \forall j \in \mathbb{N}$;

(e) $\delta_{n, j}>0, \forall n, j \in \mathbb{N}$ and $\sum_{j=1}^{\infty} \delta_{n, j}=1, \forall n \in \mathbb{N}$.

Let $\left\{x_{n}\right\}_{n \in \mathbb{N}}$ be a sequence generated by

$$
\left\{\begin{array}{l}
x_{1} \in H_{1} \quad \text { chosen arbitrarily, } \\
w_{n}=\beta_{n, 0} x_{n}+\sum_{j=1}^{\infty} \beta_{n, j} S_{j} x_{n} \\
y_{n}=w_{n}+\gamma \eta \sum_{j=1}^{\infty} \delta_{n, j} A^{*}\left(T_{j}-I\right) A\left(w_{n}\right) \\
x_{n+1}=\alpha_{n} z+\left(1-\alpha_{n}\right) y_{n}
\end{array}\right.
$$


where $\eta \in(0,1), \gamma \in\left(0, \frac{1}{\lambda \eta}\right)$, with $\lambda$ being the spectral radius of the operator $A^{*} A$. If $\Gamma:=\{x \in C: A x \in Q\} \neq \varnothing$, then the sequence $\left\{x_{n}\right\}_{n \in \mathbb{N}}$ defined in (4.1) converges strongly to $P_{C} z$, where $P_{C}$ is the metric projection from $H_{1}$ onto $C$.

Proof. We divide the proof into several steps. It is clear that $\Gamma$ is a closed and convex subset of $C$. Set

$$
u=P_{\Gamma} z
$$

Step 1. We prove that the sequences $\left\{x_{n}\right\}_{n \in \mathbb{N}},\left\{w_{n}\right\}_{n \in \mathbb{N}},\left\{y_{n}\right\}_{n \in \mathbb{N}},\left\{S_{j} x_{n}\right\}_{j, n \in \mathbb{N}}$ $\left\{A w_{n}\right\}_{n \in \mathbb{N}}$ are bounded. We first show that $\left\{x_{n}\right\}_{n \in \mathbb{N}}$ is bounded. Let $v \in \Gamma$ be arbitrarily chosen. In view of Lemma 2.6 there exists a continuous, strictly increasing and convex function $h_{3}:[0, \infty) \rightarrow[0, \infty)$ such that

$$
\begin{aligned}
& \left\|w_{n}-v\right\|^{2} \\
= & \left\|\beta_{n, 0} x_{n}+\sum_{j=1}^{\infty} \beta_{n, j} S_{j} x_{n}-v\right\|^{2} \\
\leq & \beta_{n, 0}\left\|x_{n}-v\right\|^{2}+\sum_{j=1}^{\infty} \beta_{n, j}\left\|S_{j} x_{n}-v\right\|^{2}-\beta_{n, 0} \beta_{n, j} h_{3}\left(\left\|x_{n}-S_{j} x_{n}\right\|\right) \\
\leq & \beta_{n, 0}\left\|x_{n}-v\right\|^{2}+\sum_{\substack{j=1 \\
=}} \beta_{n, j}\left\|x_{n}-v\right\|^{2}-\beta_{n, 0} \beta_{n, j} h_{3}\left(\left\|x_{n}-S_{j} x_{n}\right\|\right) \\
= & \left\|x_{n}-v\right\|^{2}-\beta_{n, 0} \beta_{n, j} h_{3}\left(\left\|x_{n}-S_{j} x_{n}\right\|\right), \quad \forall j \in \mathbb{N} .
\end{aligned}
$$

This, together with Lemma 2.2(i), implies that

$$
\begin{aligned}
\left\|y_{n}-v\right\|^{2}= & \left\|w_{n}+\gamma \eta \sum_{j=1}^{\infty} \delta_{n, j} A^{*}\left(T_{j}-I\right) A\left(w_{n}\right)-v\right\|^{2} \\
= & \left\|w_{n}-v\right\|^{2}+\gamma^{2} \eta^{2}\left\|\sum_{j=1}^{\infty} \delta_{n, j} A^{*}\left(T_{j}-I\right) A\left(w_{n}\right)\right\|^{2} \\
& +2 \gamma \eta\left\langle w_{n}-v, \sum_{j=1}^{\infty} \delta_{n, j} A^{*}\left(T_{j}-I\right) A\left(w_{n}\right)\right\rangle \\
\leq & \left\|w_{n}-v\right\|^{2}+\gamma^{2} \eta^{2} \sum_{j=1}^{\infty} \delta_{n, j}\left\|A^{*}\left(T_{j}-I\right) A\left(w_{n}\right)\right\|^{2} \\
& +2 \gamma \eta\left\langle w_{n}-v, \sum_{j=1}^{\infty} \delta_{n, j} A^{*}\left(T_{j}-I\right) A\left(w_{n}\right)\right\rangle \\
\leq & \left\|x_{n}-v\right\|^{2}+\gamma^{2} \eta^{2} \sum_{j=1}^{\infty} \delta_{n, j}\left\langle\left(T_{j}-I\right) A\left(w_{n}\right), A A^{*}\left(T_{j}-I\right) A\left(w_{n}\right)\right\rangle \\
& +2 \gamma \eta \sum_{j=1}^{\infty} \delta_{n, j}\left\langle w_{n}-v, A^{*}\left(T_{j}-I\right) A\left(w_{n}\right)\right\rangle \\
& -\beta_{n, 0} \beta_{n, j} h_{3}\left(\left\|x_{n}-S_{j} x_{n}\right\|\right), \quad \forall j \in \mathbb{N} .
\end{aligned}
$$


From the definition of $\lambda$, we conclude that

$$
\begin{aligned}
& \gamma^{2} \eta^{2} \sum_{j=1}^{\infty} \delta_{n, j}\left\langle\left(T_{j}-I\right) A\left(w_{n}\right), A A^{*}\left(T_{j}-I\right) A\left(w_{n}\right)\right\rangle \\
\leq & \lambda \gamma^{2} \eta^{2} \sum_{j=1}^{\infty} \delta_{n, j}\left\langle\left(T_{j}-I\right) A\left(w_{n}\right),\left(T_{j}-I\right) A\left(w_{n}\right)\right\rangle \\
= & \lambda \gamma^{2} \eta^{2} \sum_{j=1}^{\infty} \delta_{n, j}\left\|\left(T_{j}-I\right) A\left(w_{n}\right)\right\|^{2} .
\end{aligned}
$$

Let $\pi_{n}:=2 \gamma \eta \sum_{j=1}^{\infty} \delta_{n, j}\left\langle w_{n}-v, A^{*}\left(T_{j}-I\right) A\left(w_{n}\right)\right\rangle$. Then in view of Lemma 2.3(i), we deduce that

$$
\begin{aligned}
\pi_{n}= & 2 \gamma \eta \sum_{j=1}^{\infty} \delta_{n, j}\left\langle A\left(w_{n}-v\right),\left(T_{j}-I\right) A\left(w_{n}\right)\right\rangle \\
= & 2 \gamma \eta \sum_{j=1}^{\infty} \delta_{n, j}\left\langle A\left(w_{n}-v\right)+\left(T_{j}-I\right) A\left(w_{n}\right)\right. \\
& \left.\left.-\left(T_{j}-I\right) A\left(w_{n}\right), T_{j}-I\right) A\left(w_{n}\right)\right\rangle \\
= & 2 \gamma \eta \sum_{j=1}^{\infty} \delta_{n, j}\left\langle T_{j}\left(A\left(w_{n}\right)\right)-A v,\left(T_{j}-I\right) A\left(w_{n}\right)\right\rangle-\left\|\left(T_{j}-I\right) A\left(w_{n}\right)\right\|^{2} \\
\leq & 2 \gamma \eta \sum_{j=1}^{\infty} \delta_{n, j}\left(\frac{1}{2}\left\|\left(T_{j}-I\right) A\left(w_{n}\right)\right\|^{2}-\left\|\left(T_{j}-I\right) A\left(w_{n}\right)\right\|^{2}\right) \\
\leq & -\gamma \eta \sum_{j=1}^{\infty} \delta_{n, j}\left\|\left(T_{j}-I\right) A\left(w_{n}\right)\right\|^{2} .
\end{aligned}
$$

This, together with (4.3), implies that

$$
\begin{aligned}
\left\|y_{n}-v\right\|^{2} \leq & \left\|x_{n}-v\right\|^{2}-\gamma \eta(1-\lambda \gamma \eta) \sum_{j=1}^{\infty} \delta_{n, j}\left\|\left(T_{j}-I\right) A\left(w_{n}\right)\right\|^{2} \\
& -\beta_{n, 0} \beta_{n, j} h_{3}\left(\left\|x_{n}-S_{j} x_{n}\right\|\right), \quad \forall j \in \mathbb{N} .
\end{aligned}
$$

In view of (4.1) and (4.6) we conclude that

$$
\begin{aligned}
& \left\|x_{n+1}-u\right\|^{2} \\
= & \left\|\alpha_{n} z+\left(1-\alpha_{n}\right) y_{n}-u\right\|^{2} \\
\leq & \alpha_{n}\|z-u\|^{2}+\left(1-\alpha_{n}\right)\left\|y_{n}-u\right\|^{2} \\
\leq & \alpha_{n}\|z-u\|^{2} \\
& +\left(1-\alpha_{n}\right)\left[\left\|x_{n}-u\right\|^{2}-\gamma \eta(1-\lambda \gamma \eta) \sum_{j=1}^{\infty} \delta_{n, j}\left\|\left(T_{j}-I\right) A\left(w_{n}\right)\right\|^{2}\right. \\
& \left.-\beta_{n, 0} \beta_{n, j} h_{3}\left(\left\|x_{n}-S_{j} x_{n}\right\|\right)\right], \quad \forall j \in \mathbb{N} .
\end{aligned}
$$


This implies that

$$
\begin{aligned}
\left\|x_{n+1}-v\right\| & =\left\|\alpha_{n} z+\left(1-\alpha_{n}\right) y_{n}-v\right\| \\
& \leq \alpha_{n}\|z-v\|+\left(1-\alpha_{n}\right)\left\|y_{n}-v\right\| \\
& \leq \alpha_{n}\|z-v\|+\left(1-\alpha_{n}\right)\left\|x_{n}-v\right\| \\
& \leq \max \left\{\|z-v\|,\left\|x_{n}-v\right\|\right\} .
\end{aligned}
$$

By induction, we obtain

$$
\left\|x_{n+1}-v\right\| \leq \max \left\{\|z-v\|,\left\|x_{1}-v\right\|\right\}
$$

for all $n \in \mathbb{N}$. This implies that the sequence $\left\{\left\|x_{n}-v\right\|\right\}_{n \in \mathbb{N}}$ is bounded and hence the sequence $\left\{x_{n}\right\}_{n \in \mathbb{N}}$ is bounded. This, together with (4.1), implies that the sequences $\left\{y_{n}\right\}_{n \in \mathbb{N}},\left\{w_{n}\right\}_{n \in \mathbb{N}},\left\{A w_{n}\right\}_{n \in \mathbb{N}}$ and $\left\{S_{j} x_{n}\right\}_{j, n \in \mathbb{N}}$ are bounded too. Let

$$
\begin{aligned}
M_{5}:= & \sup \left\{\mid\|z-u\|^{2}-\left\|x_{n}-u\right\|^{2}\right. \\
& +\gamma \eta(1-\lambda \gamma \eta) \sum_{j=1}^{\infty} \delta_{n, j}\left\|\left(T_{j}-I\right) A\left(w_{n}\right)\right\|^{2} \\
& \left.+\beta_{n, 0} \beta_{n, j} h_{3}\left(\left\|x_{n}-S_{j} x_{n}\right\|\right): j, n \in \mathbb{N}\right\} .
\end{aligned}
$$

It follows from (4.7) that

$$
\begin{aligned}
& \beta_{n, 0} \beta_{n, j} h_{3}\left(\left\|x_{n}-S_{j} x_{n}\right\|\right)+\gamma \eta(1-\lambda \gamma \eta) \sum_{j=1}^{\infty} \delta_{n, j}\left\|\left(T_{j}-I\right) A\left(w_{n}\right)\right\|^{2} \\
\leq & \left\|x_{n}-u\right\|^{2}-\left\|x_{n+1}-u\right\|^{2}+\alpha_{n} M_{5}, \quad \forall j \in \mathbb{N} .
\end{aligned}
$$

Step 2. We prove that for any $n \in \mathbb{N}$

$$
\left\|x_{n+1}-u\right\|^{2} \leq\left(1-\alpha_{n}\right)\left\|x_{n}-u\right\|^{2}+2 \alpha_{n}\left\langle x_{n+1}-u, z-u\right\rangle .
$$

Let us show (4.9). In view of Lemma 2.2(iii) and (4.1), we obtain

$$
\begin{aligned}
\left\|x_{n+1}-u\right\|^{2} & =\left\|\alpha_{n} z+\left(1-\alpha_{n}\right) y_{n}-u\right\|^{2} \\
& \leq\left\|\left(1-\alpha_{n}\right)\left(y_{n}-u\right)\right\|^{2}+2 \alpha_{n}\left\langle x_{n+1}-u, z-u\right\rangle \\
& \leq\left(1-\alpha_{n}\right)\left\|y_{n}-u\right\|^{2}+2 \alpha_{n}\left\langle x_{n+1}-u, z-u\right\rangle \\
& \leq\left(1-\alpha_{n}\right)\left\|x_{n}-u\right\|^{2}+2 \alpha_{n}\left\langle x_{n+1}-u, z-u\right\rangle .
\end{aligned}
$$

Step 3. We prove that $x_{n} \rightarrow u$ as $n \rightarrow \infty$.

We discuss the following two possible cases: 
Case 1. Suppose that there exists $n_{0} \in \mathbb{N}$ such that $\left\{\left\|x_{n}-u\right\|\right\}_{n=n_{0}}^{\infty}$ is nonincreasing. Then, the sequence $\left\{\left\|x_{n}-u\right\|\right\}_{n \in \mathbb{N}}$ is convergent. Thus we have $\left\|x_{n}-u\right\|^{2}-$ $\left\|x_{n+1}-u\right\|^{2} \rightarrow 0$ as $n \rightarrow \infty$. This, together with condition (d), (e) and (4.8), implies that

$$
\lim _{n \rightarrow \infty}\left\|\left(T_{j}-I\right) A\left(w_{n}\right)\right\|=0 \quad \text { and } \lim _{n \rightarrow \infty} h_{3}\left(\left\|x_{n}-S_{j} x_{n}\right\|\right), \quad \forall j \in \mathbb{N} .
$$

From the properties of $h_{3}$, we conclude that

$$
\lim _{n \rightarrow \infty}\left\|\left(T_{j}-I\right) A\left(w_{n}\right)\right\|=0 \quad \text { and } \quad \lim _{n \rightarrow \infty}\left\|x_{n}-S_{j} x_{n}\right\|, \quad \forall j \in \mathbb{N} .
$$

On the other hand, we have

$$
\begin{aligned}
y_{n}-w_{n} & =\gamma \eta \sum_{j=1}^{\infty} \delta_{n, j} A^{*}\left(T_{j}-I\right) A\left(w_{n}\right), w_{n}-x_{n} \\
& =\sum_{j=1}^{\infty} \beta_{n, j}\left(S_{j} x_{n}-x_{n}\right) \text { and } x_{n+1}-y_{n}=\alpha_{n}\left(z-y_{n}\right)
\end{aligned}
$$

This implies that

$$
\lim _{n \rightarrow \infty}\left\|y_{n}-w_{n}\right\|=0, \quad \lim _{n \rightarrow \infty}\left\|w_{n}-x_{n}\right\|=0 \quad \text { and } \quad \lim _{n \rightarrow \infty}\left\|x_{n+1}-y_{n}\right\|=0
$$

and hence

$$
\lim _{n \rightarrow \infty}\left\|x_{n+1}-x_{n}\right\|=0
$$

Since $\left\{x_{n}\right\}_{n \in \mathbb{N}}$ is bounded, there exists a subsequence $\left\{x_{n_{i}}\right\}_{i \in \mathbb{N}}$ of $\left\{x_{n}\right\}_{n \in \mathbb{N}}$ such that $x_{n_{i}} \rightarrow y \in H_{1}$, and

$$
\limsup _{n \rightarrow \infty}\left\langle x_{n}-u, z-u\right\rangle=\lim _{i \rightarrow \infty}\left\langle x_{n_{i}}-u, z-u\right\rangle=\langle y-u, z-u\rangle .
$$

In view of Lemma 2.1(i) we conclude that $y \in C$. This, together with Lemma 2.1(ii), implies that

$$
\begin{aligned}
\limsup _{n \rightarrow \infty}\left\langle x_{n+1}-u, z-u\right\rangle \leq & \limsup _{n \rightarrow \infty}\left\langle x_{n+1}-x_{n}, z-u\right\rangle \\
& +\limsup _{n \rightarrow \infty}\left\langle x_{n}-u, z-u\right\rangle \\
= & \limsup _{n \rightarrow \infty}\left\langle x_{n}-u, z-u\right\rangle \\
= & \lim _{i \rightarrow \infty}\left\langle x_{n_{i}}-u, z-u\right\rangle \\
= & \langle y-u, z-u\rangle \\
= & \left\langle y-P_{C} z, z-P_{C} z\right\rangle \\
\leq & 0 .
\end{aligned}
$$

Thus we have the desired result by (4.9) and Lemma 2.4 . 
Case 2. Suppose that there exists a subsequence $\left\{n_{i}\right\}_{i \in \mathbb{N}}$ of $\{n\}_{n \in \mathbb{N}}$ such that

$$
\left\|x_{n_{i}}-u\right\|<\left\|x_{n_{i}+1}-u\right\|
$$

for all $i \in \mathbb{N}$. Then, by Lemma 2.5, there exists a nondecreasing sequence $\left\{m_{k}\right\}_{k \in \mathbb{N}} \subset$ $\mathbb{N}$ such that $m_{k} \rightarrow \infty$,

$$
\left\|u-x_{m_{k}}\right\| \leq\left\|u-x_{m_{k}+1}\right\| \quad \text { and } \quad\left\|u-x_{k}\right\| \leq\left\|u-x_{m_{k}+1}\right\|
$$

for all $k \in \mathbb{N}$. This, together with (4.6), implies that

$$
\begin{aligned}
& \beta_{m_{k}, 0} \beta_{m_{k}, j} h_{3}\left(\left\|x_{m_{k}}-S_{j} x_{m_{k}}\right\|\right) \\
& +\gamma \eta(1-\lambda \gamma \eta) \sum_{j=1}^{\infty} \delta_{m_{k}, j}\left\|\left(T_{j}-I\right) A\left(w_{m_{k}}\right)\right\|^{2} \\
\leq & \left\|x_{m_{k}}-u\right\|^{2}-\left\|x_{m_{k}+1}-u\right\|^{2}+\alpha_{m_{k}} M_{5} \\
\leq & \alpha_{m_{k}} M_{5},
\end{aligned}
$$

for all $j, k \in \mathbb{N}$. Then, by conditions (a), (d) and (e), we get

$$
\lim _{k \rightarrow \infty}\left\|\left(T_{j}-I\right) A\left(w_{m_{k}}\right)\right\|=\lim _{k \rightarrow \infty} h_{3}\left(\left\|x_{m_{k}}-S_{j} x_{m_{k}}\right\|\right)=0, \quad \forall j \in \mathbb{N} .
$$

From the properties of $h_{3}$, we conclude that

$$
\lim _{k \rightarrow \infty}\left\|\left(T_{j}-I\right) A\left(w_{m_{k}}\right)\right\|=\lim _{k \rightarrow \infty}\left\|x_{m_{k}}-S_{j} x_{m_{k}}\right\|=0, \quad \forall j \in \mathbb{N} .
$$

By the same argument, as in Case 1, we arrive at

$$
\limsup _{k \rightarrow \infty}\left\langle x_{m_{k}+1}-u, z-u\right\rangle \leq 0
$$

It follows from (4.9) that

$$
\left\|x_{m_{k}+1}-u\right\|^{2} \leq\left(1-\alpha_{m_{k}}\right)\left\|x_{m_{k}}-u\right\|^{2}+2 \alpha_{m_{k}}\left\langle x_{m_{k}+1}-u, z-u\right\rangle .
$$

Since $\left\|x_{m_{k}}-u\right\| \leq\left\|x_{m_{k}+1}-u\right\|$, we have that

$$
\begin{aligned}
\alpha_{m_{k}}\left\|x_{m_{k}}-u\right\|^{2} & \leq\left\|x_{m_{k}}-u\right\|^{2}-\left\|x_{m_{k}+1}-u\right\|^{2}+2 \alpha_{m_{k}}\left\langle x_{m_{k}+1}-u, z-u\right\rangle \\
& \leq 2 \alpha_{m_{k}}\left\langle x_{m_{k}+1}-u, z-u\right\rangle .
\end{aligned}
$$

In particular, since $\alpha_{m_{k}}>0$, we obtain

$$
\left\|x_{m_{k}}-u\right\|^{2} \leq 2\left\langle x_{m_{k}+1}-u, z-u\right\rangle .
$$


In view of (4.15), we deduce that

$$
\lim _{k \rightarrow \infty}\left\|x_{m_{k}}-u\right\|=0 .
$$

This, together with (4.16), implies that

$$
\lim _{k \rightarrow \infty}\left\|x_{m_{k}+1}-u\right\|=0 .
$$

On the other hand, we have $\left\|x_{k}-u\right\| \leq\left\|x_{m_{k}+1}-u\right\|$ for all $k \in \mathbb{N}$ which implies that $x_{k} \rightarrow u$ as $k \rightarrow \infty$. Thus, we have $x_{n} \rightarrow u$ as $n \rightarrow \infty$ and hence $w_{n} \rightarrow u$ as $n \rightarrow \infty$. It follows from (4.10), (4.11), (4.14) and demiclosedness of $T_{j}-I$ at 0 that

$$
T_{j}(A u)=A u, \quad \forall j \in \mathbb{N} .
$$

This implies that $A u \in Q$, which completes the proof.

In this section our interest is in the study of the strong convergence of a Halperntype iterative algorithm for solving the following convex feasibility problem which is called split convex feasibility problem:

Find $x \in C, y \in Q \quad$ such that $A x=B y$ and $\Lambda y \in D$,

where $H_{1}, H_{2}, H_{3}$ are real Hilbert spaces, $C \subset H_{1}, Q \subset H_{2}, D \subset H_{3}$ are nonempty, closed and convex sets, and $A: H_{1} \rightarrow H_{3}, B: H_{2} \rightarrow H_{3}, \Lambda: H_{2} \rightarrow H_{3}$ are bounded linear operators. In particular, we can prove the following strong convergence theorem concerning infinite-sets split convex feasibility problems whose proof will be omitted since it can be proved by similar argument as those in Theorems 3.1, 3.2 and 4.1.

Theorem 4.2. Let $H_{1}, H_{2}$ and $H_{3}$ be real Hilbert spaces, $C \subset H_{1}, Q \subset H_{2}$, $D \subset H_{3}$ be nonempty, closed and convex sets such that

$$
C=\left\{x \in H_{1}: f(x) \leq 0\right\} \quad \text { and } \quad Q=\left\{y \in H_{2}: g(y) \leq 0\right\},
$$

where $f: H_{1} \rightarrow \mathbb{R}$ and $g: H_{2} \rightarrow \mathbb{R}$ are two convex functions which are subdifferentiable on $C$ and $Q$ respectively and their subdifferentials are bounded on bounded sets. Let $A: H_{1} \rightarrow H_{3}, B: H_{2} \rightarrow H_{3}, \Lambda: H_{2} \rightarrow H_{3}$ be bounded linear operators and $\left\{T_{j}\right\}_{j \in \mathbb{N}}$ be an infinite family of quasi-nonexpansive mappings from $\mathrm{H}_{3}$ into itself such that $\cap_{j=1}^{\infty} F\left(T_{j}\right)=D$. Assume that for each $j \in \mathbb{N}, T_{j}-I$ is demiclosed at 0 . Assume $\zeta \in(0,1), \gamma \in\left(0, \min \left\{\frac{1}{\|A\|^{2}}, \frac{1}{\|B\|^{2}}\right\}\right)$. Let $(u, w) \in H_{1} \times H_{2}$ be fixed. Let $\left\{\alpha_{n}\right\}_{n \in \mathbb{N} \cup\{0\}}$ and $\left\{\delta_{n, j}\right\}_{n, j \in \mathbb{N}}$ be sequences in $[0,1]$ satisfying the following control conditions:

(a) $\lim _{n \rightarrow \infty} \alpha_{n}=0$;

(b) $\sum_{n=0}^{\infty} \alpha_{n}=\infty$; 
(c) $\delta_{n, j}>0, \forall n, j \in \mathbb{N}$ and $\sum_{j=1}^{\infty} \delta_{n, j}=1 \forall n \in \mathbb{N}$.

Let $\left\{x_{n}\right\}_{n \in \mathbb{N} \cup\{0\}}$ be a sequence generated by

$$
\left\{\begin{array}{l}
x_{0} \in H_{1}, y_{0} \in H_{2} \quad \text { chosen arbitrarily } \\
u_{n}=P_{C_{n}}\left(x_{n}-\gamma A^{*}\left(A x_{n}-B y_{n}\right)\right) \\
z_{n}=\zeta x_{n}+(1-\zeta) u_{n} \\
s_{n}=y_{n}+\sigma \eta \sum_{j=1}^{\infty} \delta_{n, j} \Lambda^{*}\left(T_{j}-I\right) \Lambda\left(y_{n}\right) \\
w_{n}=P_{Q_{n}}\left(y_{n}+\gamma B^{*}\left(A x_{n+1}-B y_{n}\right)\right) \\
t_{n}=\zeta s_{n}+(1-\zeta) w_{n} \\
x_{n+1}=\alpha_{n} u+\left(1-\alpha_{n}\right) z_{n} \\
y_{n+1}=\alpha_{n} w+\left(1-\alpha_{n}\right) t_{n}
\end{array}\right.
$$

where $C_{n}=\left\{x \in H_{1}: f\left(x_{n}\right)+\left\langle\xi_{n}, x-x_{n}\right\rangle \leq 0\right\}, \xi_{n} \in \partial f\left(x_{n}\right), Q_{n}=\left\{y \in H_{2}\right.$ : $\left.g\left(y_{n}\right)+\left\langle\eta_{n}, y-y_{n}\right\rangle \leq 0\right\}, \eta_{n} \in \partial g\left(y_{n}\right)$ and $\eta \in(0,1), \sigma \in\left(0, \frac{1}{\lambda \eta}\right)$, with $\lambda$ being the spectral radius of the operator $\Lambda^{*} \Lambda$. If $\Omega:=\{(x, y) \in C \times Q: A x=B y, \Lambda y \in$ $D)\} \neq \varnothing$, then the sequence $\left\{\left(x_{n}, y_{n}\right)\right\}_{n \in \mathbb{N}}$ defined in (4.18) converges strongly to $P_{\Omega}(u, w)$, where $P_{\Omega}$ is the metric projection from $H_{1} \times H_{2}$ onto $\Omega$.

Considering a single operator as a special case, we obtain the following corollary directly.

Corollary 4.1. Let $H_{1}, H_{2}$ and $H_{3}$ be real Hilbert spaces, $C \subset H_{1}, Q \subset H_{2}$, $D \subset H_{3}$ be nonempty, closed and convex sets such that

$$
C=\left\{x \in H_{1}: f(x) \leq 0\right\} \quad \text { and } \quad Q=\left\{y \in H_{2}: g(y) \leq 0\right\},
$$

where $f: H_{1} \rightarrow \mathbb{R}$ and $g: H_{2} \rightarrow \mathbb{R}$ are two convex functions which are subdifferentiable on $C$ and $Q$ respectively and their subdifferentials are bounded on bounded sets. Let $A: H_{1} \rightarrow H_{3}, B: H_{2} \rightarrow H_{3}, \Lambda: H_{2} \rightarrow H_{3}$ be bounded linear operators and $\mathrm{T}: \mathrm{H}_{3} \rightarrow \mathrm{H}_{3}$ be a quasi-nonexpansive mapping such that $F(T)=D$. Assume that $T-I$ is demiclosed at 0 . Assume $\zeta \in(0,1), \gamma \in\left(0, \min \left\{\frac{1}{\|A\|^{2}}, \frac{1}{\|B\|^{2}}\right\}\right)$. Let $(u, w) \in H_{1} \times H_{2}$ be fixed. Let $\left\{\alpha_{n}\right\}_{n \in \mathbb{N} \cup\{0\}}$ be a sequence in $[0,1]$ satisfying the following control conditions:

(a) $\lim _{n \rightarrow \infty} \alpha_{n}=0$;

(b) $\sum_{n=0}^{\infty} \alpha_{n}=\infty$.

Let $\left\{x_{n}\right\}_{n \in \mathbb{N} \cup\{0\}}$ be a sequence generated by 


$$
\left\{\begin{array}{l}
x_{0} \in H_{1}, y_{0} \in H_{2} \quad \text { chosen arbitrarily, } \\
u_{n}=P_{C_{n}}\left(x_{n}-\gamma A^{*}\left(A x_{n}-B y_{n}\right)\right) \\
z_{n}=\zeta x_{n}+(1-\zeta) u_{n} \\
s_{n}=y_{n}+\sigma \eta \Lambda^{*}(T-I) \Lambda\left(y_{n}\right) \\
w_{n}=P_{Q_{n}}\left(y_{n}+\gamma B^{*}\left(A x_{n+1}-B y_{n}\right)\right) \\
t_{n}=\zeta s_{n}+(1-\zeta) w_{n} \\
x_{n+1}=\alpha_{n} u+\left(1-\alpha_{n}\right) z_{n} \\
y_{n+1}=\alpha_{n} w+\left(1-\alpha_{n}\right) t_{n}
\end{array}\right.
$$

where $C_{n}=\left\{x \in H_{1}: f\left(x_{n}\right)+\left\langle\xi_{n}, x-x_{n}\right\rangle \leq 0\right\}, \xi_{n} \in \partial f\left(x_{n}\right), Q_{n}=\left\{y \in H_{2}\right.$ : $\left.g\left(y_{n}\right)+\left\langle\eta_{n}, y-y_{n}\right\rangle \leq 0\right\}, \eta_{n} \in \partial g\left(y_{n}\right)$ and $\eta \in(0,1), \sigma \in\left(0, \frac{1}{\lambda \eta}\right)$, with $\lambda$ being the spectral radius of the operator $\Lambda^{*} \Lambda$. If $\Omega:=\{(x, y) \in C \times Q: A x=B y, \Lambda y \in$ $D)\} \neq \varnothing$, then the sequence $\left\{\left(x_{n}, y_{n}\right)\right\}_{n \in \mathbb{N}}$ converges strongly to $P_{\Omega}(u, w)$, where $P_{\Omega}$ is the metric projection from $H_{1} \times H_{2}$ onto $\Omega$.

\section{Remark 4.1.}

(1) We extend the main result of [31] from two quasi-nonexpansive mappings to a countable family of quasi-nonexpansive mappings; furthermore, our method of proof is different from that in [31].

(2) Our strongly iterative sequence (4.1) is of modified Halpern-type scheme. In [31], the author studied a modified Mann-type scheme with weak convergence for split common fixed-point problems in Hilbert spaces; they are independent of each other.

(3) In most cases strong convergence is more desirable than weak convergence.

\section{CONCLUDING REMARKS}

In this paper, we study the convex feasibility problem (1.1) in Hilbert spaces. We introduce iterative algorithm (3.1) and obtain a strongly convergent sequence of Halpern-type to a solution of the problem and provide an affirmative answer to question 1.1 and also obtain some strong convergence theorems. We end this section with the following question:

Is there any strongly convergent iterative sequence of Halpern-type to a solution of the following convex feasibility problem in the consistent case?

find $h_{1} \in C_{1}, h_{2} \in C_{2}, \ldots, h_{m-1} \in C_{m-1}$ such that $A_{1} h_{1}=A_{2} h_{2}=\ldots=A_{m-1} h_{m-1}$,

where $m \geq 4$ is a natural number, $H_{1}, H_{2}, \ldots, H_{m}$ are real Hilbert spaces, $C_{i} \subset H_{i}$ (i=1,2,..,m-1) are nonempty, closed and convex sets, and $A_{i}: H_{i} \rightarrow H_{m}(\mathrm{i}=1,2, \ldots, \mathrm{m}-1)$ are bounded linear operators. 


\section{ACKNOWLEDGMENTS}

The author would like to thank the editor and the referees for sincere evaluation and constructive comments which improved the paper considerably. This work was conducted with a postdoctoral fellowship at the National Sun Yat-sen University of Kaohsiung, Taiwan.

\section{REFERENCES}

1. A. Aleyner and S. Reich, Block-iterative algorithms for solving convex feasibility problems in Hilbert and in Banach, Journal of Mathematical Analysis and Applications, 343(1) (2008), 427-435.

2. H. Attouch, J. Bolte, P. Redont and A. Soubeyran, Alternating proximal algorithms for weakly coupled minimization problems, Applications to dynamical games and PDEs, Journal of Convex Analysis, 15 (2008), 485-506.

3. H. H. Bauschke and J. M. Borwein, On projection algorithms for solving convex feasibility problems, SIAM Review, 38(3) (1996), 367-426.

4. H. H. Bauschke and P. L. Combettes, A weak-to-strong convergence principle for Fejrmonotone methods in Hilbert spaces, Math. Oper. Res., 26(2) (2001), 248-264.

5. Ch. Byrne, Iterative oblique projection onto convex sets and the split feasibility problem, Inverse Problems, 18 (2002), 441-453.

6. C. Byrne, A Unified Treatment of Some Iterative Algorithms in Signal Processing and Image Reconstruction, Marcel Dekker, New York, 1984.

7. L. C. Ceng, Q. H. Ansari and J. C. Yao, Relaxed extragradient methods for finding minimum-norm solutions of the split feasibility problem, Nonlinear Analysis, 75 (2012), 2116-2125.

8. L. C. Ceng, Q. H. Ansari and J. C. Yao, An extragradient method for solving split feasibility and fixed point problems, Computers and Mathematics with Applications, 64 (2012), 633-642.

9. L. C. Ceng, Q. H. Ansari and C. F. Wen, Implicit relaxed and hybrid methods with regularization for minimization problems and asymptotically strict pseudocontractive mappins in the intermediate sense, Abstract and Applied Analysis, 2013 (2013), Article ID 854297, 14 pp.

10. L. C. Ceng, Q. H. Ansari and J. C. Yao, Mann type iterative methods for finding a common solution of split feasibility and fixed point problems, Positivity, 16 (2012), 471-495.

11. L. C. Ceng, H. Gupta and C. F. Wen, The Mann-type extragradient iterative algorithms with regularization for solving variational inequality problems, split feasibility, and fixed point problems, Abstract and Applied Analysis, 2013 (2013), Article ID 378750, pp. 19 
12. L. C. Ceng, Q. H. Ansari and J. C. Yao, Extragradient-projection method for solving constrained convex minimization problems, Numerical Algebra, Control and Optimization, 1 (2011), 341-359.

13. L. C. Ceng, Q. H. Ansari and J. C. Yao, Some iterative methods for finding fixed points and for solving constrained convex minimization problems, Nonlinear Analysis, 74 (2011), 5286-5302.

14. L. C. Ceng, Q. H. Ansari and C. F. Wen, Multi-step implicit iterative methods with regularization for minimization problems and fixed point problems, Journal of Inequalities and Applications, 2013 2013:240, 26 pp. doi:10.1168/1029-242X-2013-240.

15. L. C. Ceng, H. K. Xu and C. F. Wen, Relaxed viscosity approximation methods with regularization for constrained minimization problems, Journal of Applied Mathematics, 2013 (2013), Article ID 531859, 19 pp.

16. L. C. Ceng, A. Petrusel and J. C. Yao, Relaxed extragradient methods with regularization for general system of variational inequalities with constraints of split feasibility and fixed point problems, Abstract and Applied Analysis, 2013 (2013), Article ID 891232, 25 pp.

17. L. C. Ceng and C. F. Wen, Hybrid gradient-projection algorithm for solving constrained convex minimization problems with generalized mixed equilibrium problems, Journal of Function Spaces and Applications, 2012 (2012), Article ID 678353, 26 pp.

18. Y. Censor, T. Bortfeld, B. Martin and A. Trofimov, A unified approach for inversion problems in intensity-modulated radiation therapy, Physics in Medicine and Biology, $\mathbf{5 1}$ (2006), 2353-2365.

19. Y. Censor and T. Elfving, A multiprojection algorithm using Bregman projections in a product space, Numerical Algorithms, 8 (1994), 221-239.

20. Y. Censor, T. Elfving, N. Kopf and T. Bortfled, The multi-sets split feasibility problem and it applications to inverse problems, Inverse Problems, 21 (2005), 2071-2084.

21. Y. Censor and A. Segal, The split common fixed point problem for directed operators, J. Convex Anal., 16 (2009), 587-600.

22. S. S. Chang, J. K. Kim and X. R. Wang, Modified block iterative algorithm for solving convex feasibility problems in Banach spaces, J. Inequal. Appl. V., 2010 (2010), 14 pp., doi:10.1155/2010/86968 (Article ID 869684).

23. F. Deutsch, Best Approximation in Inner Product Spaces, Springer-Verlag, New York, 2001.

24. K. Geobel and W. A. Kirk, Topics on Metric Fixed Point Theory, in: Cambridge Studies in Advanced Mathematics, Vol. 28, Cambridge University Press, 1990.

25. B. Halpern, Fixed points of nonexpanding mappings, Bull. Amer. Math. Soc., 73 (1967), 957-961.

26. Z. R. Kong, L. C. Ceng and C. F. Wen, Some modified extragradient methods for solving split feasibility and fixed point problems, Abstract and Applied Analysis, 2012 (2012), Article ID 975981, 32 pp. 
27. P. E. Mainge, The viscosity approximation process for quasi-nonexpansive mappings in Hilbert spaces, Comput. Math. Appl., 59(1) (2010), 74-79.

28. P. E. Maingé, Strong convergence of projected subgradient methods for nonsmooth and nonstrictly convex minimization, Set-Valued Anal., 16 (2008), 899-912.

29. S. Maruster and C. Popirlan, On the Mann-type iteration and convex feasibility problem, J. Comput. Appl. Math., 212 (2008), 390-396.

30. E. Masad and S. Reich, A note on the multiple-set split convex feasibility problem in Hilbert space, Journal of Nonlinear and Convex Analysis, 8 (2007), 367-371.

31. A. Moudafi, A note on the split common fixed-point problems for quasi-nonexpansive operators, Nonlinea Analysis, 74 (2011), 4083-4087.

32. Abdellatif Moudafi, A relaxed alternating CQ-algorithm for convex feasibility problems, Nonlinea Analysis, 79 (2013), 117-121.

33. Weerayuth Nilsrakoo and Satit Saejung, Strong convegence theorems by Halpern-Mann iterations for relatively nonexpansive mappings in Banach spaces, Applied Mathematics and Computation, 217 (2011), 6577-6586.

34. S. Reich, Approximating fixed points of nonexpanding mappings, Panamer Math. J., 4 (1994), 23-28.

35. N. Shoiji and W. Takahashi, Strong convergence of approxiamted sequences for nonexpansive mappings in Banach spaces, Proc. Math. Soc., 125 (1997), 3641-3645.

36. Y. Song and X. Chai, Halpern iteration for firmly type nonexpansive mappings, Nonlinear Anal., 71 (2009), 4500-4506.

37. W. Takahashi, Nonlinear Functional Analysis, Fixed Point Theory and Its Applications, Yokahama Publishers, Yokahama, 2000.

38. W. Takahashi and G. E. Kim, Approximating fixed points of nonexpansive mappings in Banach spaces, Math. Japan., 48 (1998), 1-9.

39. R. Wittmann, Approximation of fixed points of nonexpansive mappings, Arch. Math., 58 (1992), 486-491.

40. H. K. Xu, A variable Krasnoseĺskii-Mann algorithm and the multiple-set split feasibility problem, Inverse Problems, 22 (2006), 2021-2034.

41. H. K. Xu and T. K. Kim, Convergence of hybrid steepest-descent methods for variational inequalities, Journal of Optimization Theory and Applications, 119(1) (2003), 185-201.

42. I. Yamada, Sinal Processing Applications of a Pair of Simple Fixed Point Algorithms, Approximation and optimization in image restoration and reconstruction, Poquerolles, France, 2009.

43. Y. Yao, R. Chen, G. Marino and Y. C. Liou, Applications of fixed point and optimization methods to the multiple-sets split feasibility problem, Journal of Applied Mathematics, 2012 (2012), 21, Article ID 927530. 
44. J. Zhao and Q. Yang, Several solution methods for the split feasibility problem, Inverse Problems, 21 (2005), 1791-1799.

Eskandar Naraghirad

Department of Mathematics

Yasouj University

Yasouj 75918

Iran

and

Department of Applied Mathematics

National Sun Yat-Sen University

Kaohsiung 804, Taiwan

E-mail: eskandarrad@gmail.com

esnaraghirad@mail.yu.ac.ir 